\title{
Bimetallic Nickel Complexes Containing Benzotriazole Derived Diamine-Bisphenolate Ligands as Highly Active Catalysts for Copolymerization of Carbon Dioxide with Cyclohexene Oxide: Synthesis, Catalysis and Kinetics
}

Wei-Fang Tsao, Guan-Lin Liu, Yu-Chia Su, ${ }^{*}$ Chu-Chieh Lin,* and Bao-Tsan Ko*

Department of Chemistry, National Chung Hsing University, Taichung 402, Taiwan

Figure S1. Mass spectrum of dinickel complex 1 was obtained using positive electron spray ionization $\left(\mathrm{ESI}^{+}\right)$technique.

Figure S2. ORTEP drawing of complex 2 with probability ellipsoids drawn at level $30 \%$. Hydrogen atoms are omitted for clarity.

Figure S3. ORTEP drawing of complex 3 with probability ellipsoids drawn at level $20 \%$. Hydrogen atoms are omitted for clarity.

Figure S4. ORTEP drawing of complex 4 with probability ellipsoids drawn at level $20 \%$. Hydrogen atoms are omitted for clarity.

Figure S5. ORTEP drawing of complex 5 with probability ellipsoids drawn at level $20 \%$. Hydrogen atoms are omitted for clarity.

Figure S6. ORTEP drawing of complex 6 with probability ellipsoids drawn at level $30 \%$. Hydrogen atoms are omitted for clarity.

Figure S7. ORTEP drawing of complex 7 with probability ellipsoids drawn at level $30 \%$. Hydrogen atoms are omitted for clarity.

Figure S8. The plot of Mn (घ) and PDI ( $\mathbf{\Delta})$ (determined from GPC analysis) versus CHO conversion for $\mathrm{CO}_{2}$-copolymerization of $\mathrm{CHO}$ using dinickel complex $\mathbf{1}$ as the catalyst $\left([\mathrm{CHO}]_{0} /[\mathbf{1}]_{0}=3200\right)$ at $140{ }^{\circ} \mathrm{C}$ and $300 \mathrm{psi} \mathrm{CO}$.

Figure S9. GPC traces for the generated PCHC with a bimodal molecular weight distribution 
catalyzed by dinickel complex 1 (Table 2, entry18).

Figure S10. GPC traces for the obtained PCHC polyol with a unimodal molecular weight distribution catalyzed by dinickel complex $\mathbf{1}$ on the addition of $\mathrm{H}_{2} \mathrm{O}$ as the CTA (Table 3, entry 2).

Figure S11. ${ }^{1} \mathrm{H}$ NMR spectrum of the purified copolymer generated by using dinickel complex 1 on the addition of $\mathrm{H}_{2} \mathrm{O}$ (Table 3, entry 4) in $\mathrm{CDCl}_{3}$. The peak at $\delta 4.65 \mathrm{ppm}$ is assigned to the methine protons of repeated units in PCHC as well as the peaks at $\delta 3.56$ and 4.37 are assigned to methine protons that is adjacent to end-capped hydroxyl and carbonate in PCHC, respectively.

Figure S12. The plot of TOFs with varying initial $\mathrm{CO}_{2}$ pressures employing dinickel complex $1(0.03125 \mathrm{~mol} \%)$ at $140{ }^{\circ} \mathrm{C}$ for $1 \mathrm{~h}$.

Figure S13. A linear plot of $\ln k_{\mathrm{obs}}$ versus $\ln [1]_{0}$ exhibiting a first-order dependence on dinickel catalyst concentration. All polymerizations were performed at $140{ }^{\circ} \mathrm{C}$ and an initial $\mathrm{CO}_{2}$ pressure of 300 psi.

Table S1. Copolymerization of $\mathrm{CO}_{2}$ and $\mathrm{CHO}$ Catalyzed by Utilizing Dinickel Complex 1 under Different Initial $\mathrm{CO}_{2}$ Pressures for $1 \mathrm{~h}$

Table S2. Kinetic Studies of $\mathrm{CO}_{2} / \mathrm{CHO}$ Copolymerization Catalyzed by Dinickel Complex 1 at Various Monomer-to-Catalyst Ratio $(2400,3200,4000 \text { and } 4800)^{a}$

Table S3. Kinetic Parameters for $\mathrm{CO}_{2} / \mathrm{CHO}$ Copolymerization Catalyzed by Dinickel Complex 1 at Different Catalyst concentrations

Table S4. Crystallographic Data of Complexes 1-9 


\section{Experimental Section}

Safety Note. Caution! Perchlorate salts of nickel complexes with organic compounds may be potentially explosive. Even a small amount of chemicals of this kind needs to be handled with great attention and extreme caution.

\section{General procedures}

Deuterated solvents were dried over 4 Å molecular sieves. Nickel(II) perchlorate hexahydrate, nickel(II) nitrate hexahydrate, 2-(2H-benzotriazol-2-yl)-4-(2,4,4-trimethylpentan-2-yl)phenol, 2-(2H-Benzotriazol-2-yl)-4-methylphenol, paraformaldehyde, N,N'-diethylethylenediamine, trifluoroacetic acid, benzoic acid, 4-methoxybenzoic acid, 4-(trifluoromethyl)benzoic acid, trimethylamine $\left(\mathrm{NEt}_{3}\right)$, ethanol $(\mathrm{EtOH})$, methanol $(\mathrm{MeOH})$, dichloromethane $\left(\mathrm{CH}_{2} \mathrm{Cl}_{2}\right)$, tetrahydrofuran (THF), diethyl ether, hexane, acetone and carbon dioxide $\left(\mathrm{CO}_{2}, 99.95 \%\right)$ were purchased and used without further purification. Cyclohexene oxide (CHO) was purified by distillation over calcium hydride $\left(\mathrm{CaH}_{2}\right)$ at least four times prior to use. ${ }^{1} \mathrm{H}$ and ${ }^{13} \mathrm{C}$ NMR spectra were recorded on a Varian Mercury-400 (400 and $100 \mathrm{MHz}$ ) spectrometer. Chemical shifts for ${ }^{1} \mathrm{H}$ and ${ }^{13} \mathrm{C}$ spectra were given in parts per million (ppm) from the peak of internal tetramethylsilane (TMS). Elemental analyses were conducted on a Heraeus CHNOS Rapid Elemental Analyzer. Mass analyses were performed by employing the positive electron spray ionization $\left(\mathrm{ESI}^{+}\right)$technique on a Thermo Finnigan TSQ Quantum mass spectrometer for newly synthesized complexes upon dissolving in $\mathrm{CH}_{2} \mathrm{Cl}_{2}$ solvent. Measurements of gel permeation chromatography (GPC) were performed on a Jasco PU-2080 plus system equipped with a RI-2031 detector employing THF (HPLC grade) as the eluent. The chromatographic column was Phenomenex Phenogel $5 \mu 103 \AA$ and the calibration curve utilized to calculate $M_{n}(\mathrm{GPC})$ was generated from polystyrene standards. The calibration curve was constructed by ten polystyrene standards, and their molecular weights are in a range of 1,580 288,000 $\mathrm{gmol}^{-1}$. The GPC results were calculated using the Scientific Information Service Corporation (SISC) chromatography data solution 3.1 edition. The MALDI-TOF mass spectra for polymers 
characterization were measured on an Ultraflex III TOF mass spectrometer. 2,5-Dihydroxybenzoic acid as the matrix was used. The samples were prepared by mixing THF solution $(10 \mathrm{mg} / \mathrm{mL})$ of poly(cyclohexene carbonate) with the matrix $(1 \mu \mathrm{L})$ together. This mixture $(1 \mu \mathrm{L})$ was then spotted into a stainless steel MALDI target plate (MTP 384 target plate) and allowed to dry at ambient temperature.

\section{Synthesis of ${ }^{1} \mathrm{~L}-\mathrm{H}_{2}$ proligand}

N,N'-Diethylethylenediamine $(6.27 \mathrm{~g}, 54.0 \mathrm{mmol})$, paraformaldehyde $(3.24 \mathrm{~g}, 108.0 \mathrm{mmol})$ and 2-(2H-benzotriazol-2-yl)-4-(2,4,4-trimethylpentan-2-yl)phenol (12.92 g, $40.0 \mathrm{mmol})$ were dissolved in ethanol $(80 \mathrm{~mL})$. The solution was heated under $80{ }^{\circ} \mathrm{C}$ for $48 \mathrm{~h}$ and then cooled to room temperature. The volatile components were removed under the rotary evaporator. The residue was extracted six times with $\mathrm{CH}_{2} \mathrm{Cl}_{2}$ and deionized water, and the organic layers were dried over $\mathrm{MgSO}_{4}$. The volatile components were removed in vacuo. The final residue was recrystallized from methanol, and the resulting precipitate was collected by filtration and dried under vacuum to give white solids. Yield: $2.36 \mathrm{~g}$ (15\%). Anal. Calc. for $\mathrm{C}_{48} \mathrm{H}_{66} \mathrm{~N}_{8} \mathrm{O}_{2}: \mathrm{N}, 14.24$, C, 73.25, H, 8.45\%, Found: N, 14.25, C, 73.15, H, 8.53\%. ${ }^{1} \mathrm{H}$ NMR $\left(\mathrm{CDCl}_{3}, \mathrm{ppm}\right): \delta 7.96$ (q, 4H, Ar- $H), 7.80(\mathrm{~d}, 2 \mathrm{H}, \operatorname{Ar}-H), 7.41(\mathrm{q}, 4 \mathrm{H}, \mathrm{Ar}-H), 7.20(\mathrm{~d}, 2 \mathrm{H}, \mathrm{Ar}-H), 3.83\left(\mathrm{~s}, 4 \mathrm{H}, \mathrm{ArCH}_{2} \mathrm{~N}\right)$, $2.75\left(\mathrm{~s}, 4 \mathrm{H}, \mathrm{N}\left(\mathrm{CH}_{2} \mathrm{CH}_{2}\right) \mathrm{N}\right), 2.56\left(\mathrm{q}, 4 \mathrm{H}, \mathrm{NCH}_{2} \mathrm{CH}_{3}\right), 1.70\left(\mathrm{~s}, 4 \mathrm{H}, \operatorname{ArC}\left(\mathrm{CH}_{3}\right)_{2} \mathrm{CH}_{2} \mathrm{C}\left(\mathrm{CH}_{3}\right)_{3}\right)$, $1.35\left(\mathrm{~s}, \quad 12 \mathrm{H}, \quad \operatorname{ArC}\left(\mathrm{CH}_{3}\right)_{2} \mathrm{CH}_{2} \mathrm{C}\left(\mathrm{CH}_{3}\right)_{3}\right), \quad 1.03 \quad\left(\mathrm{t}, \quad 6 \mathrm{H}, \quad \mathrm{NCH}_{2} \mathrm{CH}_{3}\right), \quad 0.73 \quad(\mathrm{~s}, \quad 18 \mathrm{H}$, $\left.\operatorname{ArC}\left(\mathrm{CH}_{3}\right)_{2} \mathrm{CH}_{2} \mathrm{C}\left(\mathrm{CH}_{3}\right)_{3}\right) .{ }^{13} \mathrm{C} \mathrm{NMR}\left(\mathrm{CDCl}_{3}, \mathrm{ppm}\right): \delta 148.4,144.1,141.0,128.1,127.0,126.8$, 124.5, 121.7, $118.1(A r), 56.8\left(\mathrm{ArCH}_{2} \mathrm{~N}\right), 56.4\left(\mathrm{~N}\left(\mathrm{CH}_{2} \mathrm{CH}_{2}\right) \mathrm{N}\right), 50.5\left(\mathrm{NCH}_{2} \mathrm{CH}_{3}\right), 47.7$ $\left(\mathrm{ArC}\left(\mathrm{CH}_{3}\right)_{2} \mathrm{CH}_{2}\right), \quad 38.1 \quad\left(\mathrm{ArC}\left(\mathrm{CH}_{3}\right)_{2} \mathrm{CH}_{2} \mathrm{C}\left(\mathrm{CH}_{3}\right)_{3}\right), \quad 32.4 \quad\left(\mathrm{ArC}\left(\mathrm{CH}_{3}\right)_{2} \mathrm{CH}_{2} \mathrm{C}_{(}\left(\mathrm{CH}_{3}\right)_{3}\right), \quad 31.9$ $\left(\mathrm{ArC}\left(\mathrm{CH}_{3}\right)_{2} \mathrm{CH}_{2} \mathrm{C}\left(\mathrm{CH}_{3}\right)_{3}\right), 31.6\left(\mathrm{ArC}\left(\mathrm{CH}_{3}\right)_{2} \mathrm{CH}_{2} \mathrm{C}\left(\mathrm{CH}_{3}\right)_{3}\right), 11.1\left(\mathrm{NCH}_{2} \mathrm{CH}_{3}\right)$.

\section{Synthesis of ${ }^{2} \mathrm{~L}-\mathrm{H}_{2}$ proligand}

This compound was synthesized as mentioned above for ${ }^{1} \mathrm{~L}-\mathrm{H}_{2}$, except for utilizing $\mathrm{N}, \mathrm{N}^{\prime}$-diethylethylenediamine $(5.81 \mathrm{~g}, 50.0 \mathrm{mmol})$, paraformaldehyde $(3.00 \mathrm{~g}, 100.0 \mathrm{mmol})$ and 
2-(2H-benzotriazol-2-yl)-4-methylphenol $(9.01 \mathrm{~g}, 40.0 \mathrm{mmol})$ as the reactants. The final residue was recrystallized from methanol, and then the resulting precipitate was washed by hexane at $50{ }^{\circ} \mathrm{C}$ twice. The resulting precipitate was collected by filtration and dried under vacuum to give white solids. Yield: $3.54 \mathrm{~g}$ (30\%). Anal. Calc. for $\mathrm{C}_{34} \mathrm{H}_{38} \mathrm{~N}_{8} \mathrm{O}_{2}$ : N, 18.97, C, 69.13, H, 6.48\%, Found: N, 18.47, C, 69.01, H, 6.43\%. ${ }^{1} \mathrm{H}$ NMR $\left(\mathrm{CDCl}_{3}, \mathrm{ppm}\right): \delta 7.94$ (q, 4H, $\operatorname{Ar}-H), 7.65$ (s, 2H, Ar-H), 7.41 (q, 4H, Ar-H), 6.99 (s, 2H, Ar-H), 3.78 (s, 4H, ArCH $\left.H_{2} \mathrm{~N}\right), 2.73$ (s, 4H, N( $\left.\left.\mathrm{CH}_{2} \mathrm{CH}_{2}\right) \mathrm{N}\right), 2.59$ (q, 4H, $\left.\mathrm{NCH}_{2} \mathrm{CH}_{3}\right), 2.29$ (s, 3H, Ar-CH3), 1.03 (t, 3H, $\left.\mathrm{NCH}_{2} \mathrm{CH}_{3}\right)$.

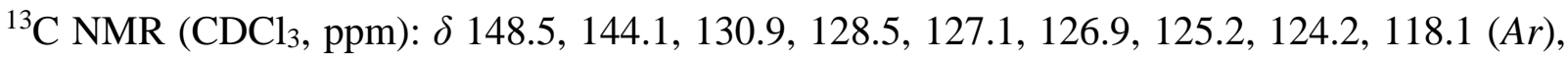
$55.9\left(\mathrm{ArCH}_{2} \mathrm{~N}\right), 50.5\left(\mathrm{~N}\left(\mathrm{CH}_{2} \mathrm{CH}_{2}\right) \mathrm{N}\right), 47.7\left(\mathrm{NCH}_{2} \mathrm{CH}_{3}\right), 20.4\left(\mathrm{Ar}-\mathrm{CH}_{3}\right), 11.0\left(\mathrm{NCH}_{2} \mathrm{CH}_{3}\right)$.

\section{Synthesis of dinickel complexes 1-9}

\section{Synthesis of complex $\left[\left({ }^{1} \mathrm{~L}\right) \mathrm{Ni}_{2}\left(\mathrm{O}_{2} \mathrm{CCF}_{3}\right)_{2}\right](1)$}

A solution of ${ }^{1} \mathrm{~L}-\mathrm{H}_{2}(0.79 \mathrm{~g}, 1.0 \mathrm{mmol})$ and nickel(II) perchlorate hexahydrate $(0.73 \mathrm{~g}, 2.0$ mmol) was dissolved in methanol $(10 \mathrm{~mL})$, and trifluoroacetic acid $(0.38 \mathrm{~mL}, 5.0 \mathrm{mmol})$ and triethylamine $(0.70 \mathrm{~mL}, 5.0 \mathrm{mmol})$ were added. The mixture was heated under reflux for $48 \mathrm{~h}$ and then cooled to ambient temperature. The volatile components were removed in vacuo. The residue was diluted with $\mathrm{CH}_{2} \mathrm{Cl}_{2}$ and extracted with a $\mathrm{NaCl}$-saturated solution. The organic layers were dried over $\mathrm{MgSO}_{4}$ and concentrated by vacuum evaporation. The final residue was washed with hexane, and the resulting precipitate was collected by filtration and dried under vacuum to give green solids. Yield: $0.90 \mathrm{~g}(80 \%)$. Anal. Calc. for $\mathrm{C}_{52} \mathrm{H}_{64} \mathrm{~F}_{6} \mathrm{~N}_{8} \mathrm{O}_{6} \mathrm{Ni}_{2}$ : N, 9.93; C, 55.34; H, 5.72\%. Found: N, 9.74; C, 55.03; H, 6.31\%. ESI-MS (m/z): 1013.6 [100\%, $\left.\left(\mathrm{M}-\mathrm{O}_{2} \mathrm{CCF}_{3}\right)^{+}\right]$.

\section{Synthesis of complex $\left[\left({ }^{2} \mathrm{~L}\right) \mathrm{Ni}_{2}\left(\mathrm{O}_{2} \mathrm{CCF}_{3}\right)_{2}\right](2)$}

This complex was prepared as mentioned above for $\mathbf{1}$, except for ${ }^{2} \mathrm{~L}-\mathrm{H}_{2}(0.59 \mathrm{~g}, 1.0 \mathrm{mmol})$ as the ligand precursor. Complex 2 as green solids were collected. Yield: $0.77 \mathrm{~g}(83 \%)$. Anal. Calc. 
for $\mathrm{C}_{38} \mathrm{H}_{36} \mathrm{~F}_{6} \mathrm{~N}_{8} \mathrm{O}_{6} \mathrm{Ni}_{2}: \mathrm{N}, 12.02 ; \mathrm{C}, 48.96 ; \mathrm{H}, 3.89 \%$. Found: N, 12.11; C, 49.34; H, 4.11\%. ESI-MS (m/z): $817.3\left[100 \%,\left(\mathrm{M}-\mathrm{O}_{2} \mathrm{CCF}_{3}\right)^{+}\right]$.

\section{Synthesis of complex $\left[\left({ }^{1} \mathrm{~L}\right) \mathrm{Ni}_{2}\left(\mathrm{O}_{2} \mathrm{CC}_{6} \mathrm{H}_{4}-4-\mathrm{CF}_{3}\right)_{2}\right](3)$}

This complex was prepared as mentioned above for $\mathbf{1}$, except for 4-(trifluoromethyl)benzoic acid (0.95 g, $5.0 \mathrm{mmol})$ as the coligand precursor. Complex $\mathbf{3}$ as green solids were collected. Yield: 1.20 g (94\%). Anal. Calc. for $\mathrm{C}_{64} \mathrm{H}_{72} \mathrm{~F}_{6} \mathrm{~N}_{8} \mathrm{O}_{6} \mathrm{Ni}_{2}: \mathrm{N}, 8.75 ; \mathrm{C}, 60.02 ; \mathrm{H}, 5.67 \%$. Found: $\mathrm{N}$, 8.34; C, 60.35; H, 5.80\%. ESI-MS (m/z): $1089.4\left[100 \%,\left\{\mathrm{M}-\left(\mathrm{O}_{2} \mathrm{CC}_{6} \mathrm{H}_{4}-4-\mathrm{CF}_{3}\right)\right\}^{+}\right]$.

\section{Synthesis of complex $\left[\left({ }^{2} \mathrm{~L}\right) \mathrm{Ni}_{2}\left(\mathrm{O}_{2} \mathrm{CC}_{6} \mathrm{H}_{4}-4-\mathrm{CF}_{3}\right)_{2}\right](4)$}

This complex was prepared as mentioned above for $\mathbf{1}$, except for ${ }^{2} \mathrm{~L}-\mathrm{H}_{2}(0.59 \mathrm{~g}, 1.0 \mathrm{mmol})$ as the ligand precursor and 4-(trifluoromethyl)benzoic acid $(0.95 \mathrm{~g}, 5.0 \mathrm{mmol})$ as the coligand precursor. Complex 4 as green solids were collected. Yield: 0.92 g (85\%). Anal. Calc. for $\mathrm{C}_{50} \mathrm{H}_{44} \mathrm{~F}_{6} \mathrm{~N}_{8} \mathrm{O}_{6} \mathrm{Ni}_{2} \cdot \mathrm{H}_{2} \mathrm{O}: \mathrm{N}, 10.17 ; \mathrm{C}, 54.48 ; \mathrm{H}, 4.21 \%$. Found: N, 9.88; C, 54.79; H, 4.28\%. ESI-MS (m/z): $893.1\left[100 \%,\left\{\mathrm{M}-\left(\mathrm{O}_{2} \mathrm{CC}_{6} \mathrm{H}_{4}-4-\mathrm{CF}_{3}\right)\right\}^{+}\right]$.

\section{Synthesis of complex $\left[\left({ }^{1} \mathrm{~L}\right) \mathrm{Ni}_{2}\left(\mathrm{O}_{2} \mathrm{CC}_{6} \mathrm{H}_{5}\right)_{2}\right](5)$}

This complex was prepared as mentioned above for $\mathbf{1}$, except for benzoic acid $(0.61 \mathrm{~g}, 5.0$ mmol) as the coligand precursor. Complex $\mathbf{5}$ as green solids were collected. Yield: $0.98 \mathrm{~g}$ (72\%). Anal. Calc. for $\mathrm{C}_{62} \mathrm{H}_{74} \mathrm{~N}_{8} \mathrm{O}_{6} \mathrm{Ni}_{2} \cdot \mathrm{H}_{2} \mathrm{O}: \mathrm{N}, 9.64 ; \mathrm{C}, 64.05 ; \mathrm{H}, 6.59 \%$. Found: $\mathrm{N}, 9.15 ; \mathrm{C}$, 64.12; H, 6.53\%. ESI-MS (m/z): $1021.3\left[100 \%,\left(\mathrm{M}-\mathrm{O}_{2} \mathrm{CC}_{6} \mathrm{H}_{5}\right)^{+}\right]$.

\section{Synthesis of complex $\left[\left({ }^{2} \mathrm{~L}\right) \mathrm{Ni}_{2}\left(\mathrm{O}_{2} \mathrm{CC}_{6} \mathrm{H}_{5}\right)_{2}\left(\mathrm{H}_{2} \mathrm{O}\right)\right](6)$}

This complex was prepared as mentioned above for $\mathbf{1}$, except for ${ }^{2} \mathrm{~L}-\mathrm{H}_{2}(0.59 \mathrm{~g}, 1.0 \mathrm{mmol})$ as the ligand precursor and benzoic acid $(0.61 \mathrm{~g}, 5.0 \mathrm{mmol})$ as the coligand precursor. Complex 6 as green solids were collected. Yield: $0.75 \mathrm{~g}(80 \%)$. Anal. Calc. for $\mathrm{C}_{48} \mathrm{H}_{48} \mathrm{~N}_{8} \mathrm{O}_{7} \mathrm{Ni}_{2} \cdot \mathrm{H}_{2} \mathrm{O}: \mathrm{N}$, 11.38; C, 58.57; H, 5.12\%. Found: N, 11.41; C, 58.51; H, 5.01\%. ESI-MS (m/z): 825.1 [100\%, 
$\left.\left(\mathrm{M}-\mathrm{O}_{2} \mathrm{CC}_{6} \mathrm{H}_{5}-\mathrm{H}_{2} \mathrm{O}\right)^{+}\right]$.

\section{Synthesis of complex $\left[\left({ }^{1} \mathrm{~L}\right) \mathrm{Ni}_{2}\left(\mathrm{O}_{2} \mathrm{CC}_{6} \mathrm{H}_{4}-4-\mathrm{OMe}\right)_{2}\left(\mathrm{H}_{2} \mathrm{O}\right)\right](7)$}

This complex was prepared as mentioned above for 1, except for 4-methoxybenzoic acid ( 0.76 $\mathrm{g}, 5.0 \mathrm{mmol}$ ) as the coligand precursor. Complex 7 as green solids were collected. Yield: $1.01 \mathrm{~g}$ (83\%). Anal. Calc. for $\mathrm{C}_{64} \mathrm{H}_{80} \mathrm{~N}_{8} \mathrm{O}_{9} \mathrm{Ni}_{2}: \mathrm{N}, 9.16 ; \mathrm{C}, 62.87 ; \mathrm{H}, 6.59 \%$. Found: N, 9.27; C, 62.64; H, 6.10\%. ESI-MS (m/z): $1051.4\left[100 \%,\left\{\mathrm{M}-\left(\mathrm{O}_{2} \mathrm{CC}_{6} \mathrm{H}_{4}-4-\mathrm{OMe}\right)-\mathrm{H}_{2} \mathrm{O}\right\}^{+}\right]$.

\section{Synthesis of complex $\left[\left({ }^{2} \mathrm{~L}\right) \mathrm{Ni}_{2}\left(\mathrm{O}_{2} \mathrm{CC}_{6} \mathrm{H}_{4}-4-\mathrm{OMe}\right)_{2}\left(\mathrm{H}_{2} \mathrm{O}\right)\right](8)$}

This complex was prepared as mentioned above for $\mathbf{1}$, except for ${ }^{2} \mathrm{~L}-\mathrm{H}_{2}(0.59 \mathrm{~g}, 1.0 \mathrm{mmol})$ as the ligand precursor and 4-methoxybenzoic acid $(0.76 \mathrm{~g}, 5.0 \mathrm{mmol})$ as the coligand precursor. Complex 8 as green solids were collected. Yield: 0.77 g (75\%). Anal. Calc. for $\mathrm{C}_{50} \mathrm{H}_{52} \mathrm{~N}_{8} \mathrm{O}_{9} \mathrm{Ni}_{2}$ : N, 10.92; C, 58.51; H, 5.11\%. Found: N, 10.90; C, 58.47; H, 5.08\%. ESI-MS (m/z): 855.1 $\left[100 \%,\left\{\mathrm{M}-\left(\mathrm{O}_{2} \mathrm{CC}_{6} \mathrm{H}_{4}-4-\mathrm{OMe}\right)-\mathrm{H}_{2} \mathrm{O}\right\}^{+}\right]$.

\section{Synthesis of complex $\left[\left({ }^{1} \mathrm{~L}\right) \mathrm{Ni}_{2}\left(\mathrm{NO}_{3}\right)_{2}\right](9)$}

A solution of nickel(II) nitrate hexahydrate $(0.58 \mathrm{~g}, 2.0 \mathrm{mmol}),{ }^{1} \mathrm{~L}-\mathrm{H}_{2}(0.79 \mathrm{~g}, 1.0 \mathrm{mmol})$ and $\mathrm{NEt}_{3}(0.70 \mathrm{~mL}, 5.0 \mathrm{mmol})$ in methanol was heated under reflux for $48 \mathrm{~h}$, during which time the formation of a green precipitate was found. The resulting precipitate was collected by filtration. The residue was washed with $n$-hexane and the resultant precipitate was collected by filtration and then dried under vacuum to obtain green solids. Yield: $0.62 \mathrm{~g}(61 \%)$. Anal. Calc. for $\mathrm{C}_{48} \mathrm{H}_{64} \mathrm{~N}_{10} \mathrm{O}_{8} \mathrm{Ni}_{2}: \mathrm{N}, 13.65 ; \mathrm{C}, 56.16 ; \mathrm{H}, 6.28 \%$. Found: N, 13.28; C, 56.65; H, 6.65\%. ESI-MS $(\mathrm{m} / \mathrm{z}): 962.5\left[100 \%,\left(\mathrm{M}-\mathrm{NO}_{3}\right)^{+}\right]$.

\section{Copolymerization of $\mathrm{CO}_{2}$ with $\mathrm{CHO}$ Catalyzed by Dinickel Complexes}

The $\mathrm{CO}_{2}$-copolymerization of $\mathrm{CHO}$ by complex 1 (entry 5, Table 2) was exemplified as a representative procedure. $\left[\left({ }^{1} \mathrm{~L}\right) \mathrm{Ni}_{2}\left(\mathrm{O}_{2} \mathrm{CCF}_{3}\right)_{2}\right](\mathbf{1})(35.2 \mathrm{mg}, 0.03125 \mathrm{mmol})$ was dissolved in 
$100.0 \mathrm{mmol}$ of neat $\mathrm{CHO}$ in a dry nitrogen atmosphere. The mixture was added to the $100 \mathrm{~mL}$ autoclave with a magnetic stir bar under $\mathrm{CO}_{2}$ atmosphere. The reactor was then charged with $\mathrm{CO}_{2}$ until the pressure of 300 psi was reached, and the stirrer was started. The copolymerization was carried out at $140{ }^{\circ} \mathrm{C}$ for $2 \mathrm{~h}$. After cooling with ice water and venting the reactor, a small aliquot of the copolymerization mixture was taken for ${ }^{1} \mathrm{H}$ NMR spectroscopic analysis. The $\mathrm{CHO}$ conversion (60\%) and the copolymerization selectivity (>99\% PCHC; >99\% carbonate linkages) were characterized by ${ }^{1} \mathrm{H}$ NMR spectroscopy. The remaining crude was further dissolved in $5 \mathrm{~mL}$ of $\mathrm{CH}_{2} \mathrm{Cl}_{2}$ and precipitated in excess $\mathrm{MeOH}$ to give yellowish solids. In order to remove metal salts, the resulting copolymer was diluted with $20 \mathrm{~mL}$ of $\mathrm{CH}_{2} \mathrm{Cl}_{2}$ and washed with aqueous $\mathrm{HCl}(4 \mathrm{~N}, 100 \mathrm{~mL})$. The organic layer was then extracted and concentrated to $5 \mathrm{~mL}$ by vacuum evaporation. Finally, the copolymer was precipitated into $\mathrm{MeOH}(150 \mathrm{~mL})$ again to yield a white solid.

\section{Immortal Copolymerization of $\mathrm{CO}_{2}$ and $\mathrm{CHO}$ Catalyzed by Dinickel Complex 1 on the Addition of Water as the Chain Transfer Agent}

The immortal $\mathrm{CO}_{2}$-copolymerization of $\mathrm{CHO}$ (Table 3 , entry 4) by using complex $\mathbf{1}$ in combination with 100 equiv. of $\mathrm{H}_{2} \mathrm{O}$ as a representative procedure was exemplified. The similar procedure was performed as mentioned above for the $\mathrm{CO}_{2}$-copolymerization of $\mathrm{CHO}$, except for the extra addition of $\mathrm{H}_{2} \mathrm{O}(0.056 \mathrm{ml}, 3.125 \mathrm{mmol})$ as the CTA. ${ }^{1} \mathrm{H}$ NMR spectroscopic studies were used to determine the $\mathrm{CHO}$ conversion $(60 \%)$ and the copolymerization selectivity (99\% PCHC; >99\% carbonate linkages). Further purification of the afforded copolymer was similar to the procedure of $\mathrm{CO}_{2}$-copolymerization of $\mathrm{CHO}$ without adding CTA.

\section{X-ray crystallographic studies}

Suitable crystals of dinuclear nickel complexes 1-9 were mounted onto glass fiber by using perfluoropolyether oil and cooled rapidly in a stream of cold nitrogen gas. Diffraction data 
were collected by using Bruker APEX2 diffractometer at $202 \mathrm{~K}, 175 \mathrm{~K}$ or $150 \mathrm{~K}$, and intensity data were collected with a combination of $\phi$ and $\omega$ scans. All the data were corrected by Lorentz and polarization effects, and the absorption correction was conducted by the program $\mathrm{APEX}^{1}$ in $\mathrm{SADABS}^{2}$. The space group was determined based on a check of the Laue symmetry and systematic absences, and was verified by utilizing the structure solution. The molecular structure was then solved and refined with SHELXTL package. ${ }^{3}$ All non-H atoms were located from successive Fourier maps, and hydrogen atoms were treated as a riding model on their parent $\mathrm{C}$ atoms. Anisotropic thermal parameters were applied for all non- $\mathrm{H}$ atoms, and fixed isotropic parameters were employed for H-atoms. Drawing of the molecular structure was executed by utilizing Oak Ridge Thermal Ellipdoid Plots (ORTEP). ${ }^{4}$ Crystallographic data of dinickel complexes 1-9 are illustrated in Table S4.

\section{References}

1 APEX2, V2010.3; Bruker AXS Inc.: 2010.

2 SADABS V2008/1, SAINT+V7.60A; Bruker AXS Inc.: 2008.

3 Sheldrick, G. M. A short history of SHELX. Acta Crystallogr., Sect. A: Found. Crystallogr. 2008, 64, 112-122.

4 Burnett, M. N.; Johnson, C. K. ORTEPIII; Oak Ridge National Laboratory: 1996; Report ORNL-6895. 
14-C8CF3 \#1-20 RT: 0.00-0.06 AV: 20 NL: 1.79E6

T: ITMS + c ESI Full ms [150.00-2000.00]

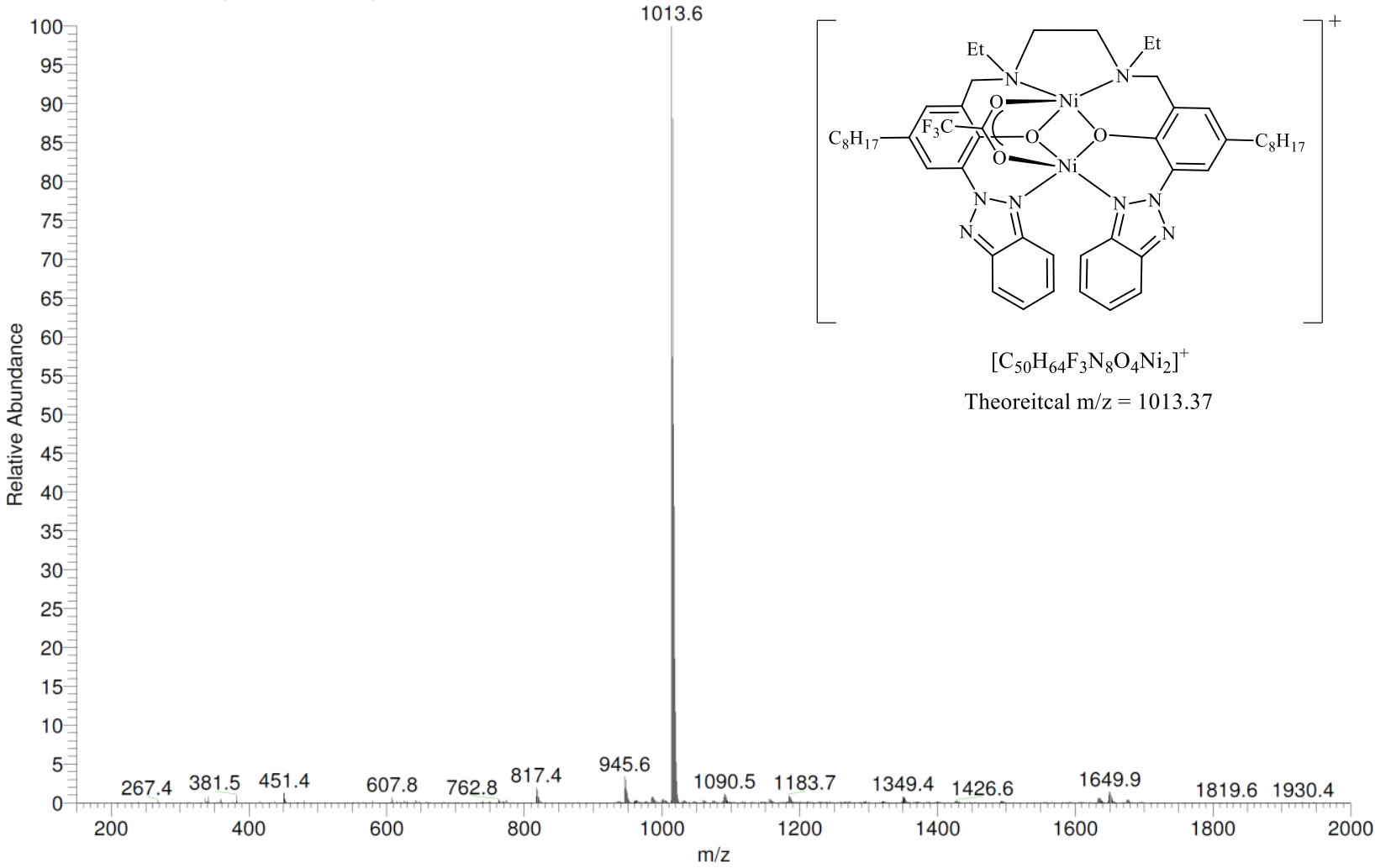

Figure S1. Mass spectrum of dinickel complex 1 was obtained using positive electron spray ionization $\left(\mathrm{ESI}^{+}\right)$technique. 


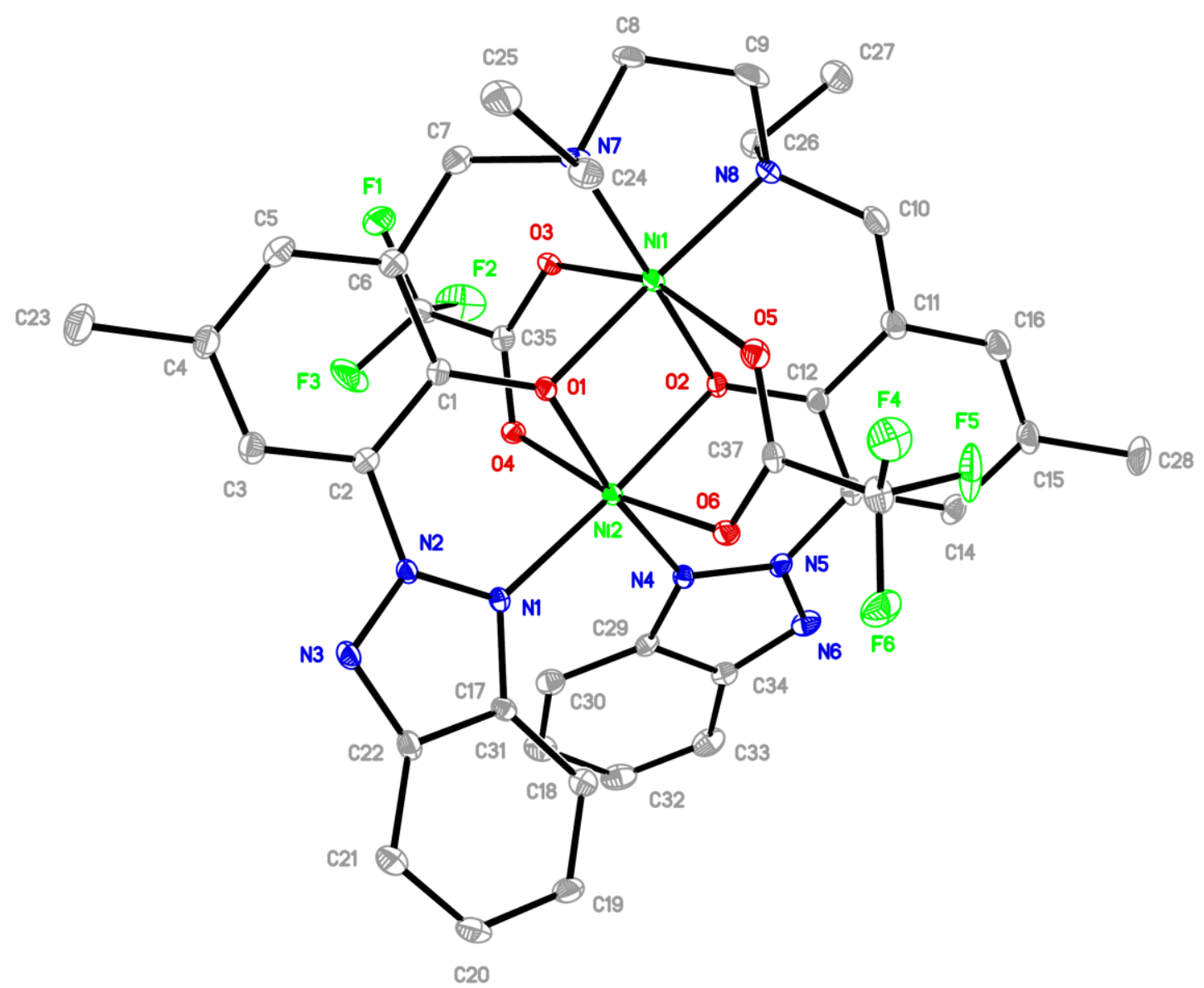

Figure S2. ORTEP drawing of complex 2 with probability ellipsoids drawn at level $30 \%$. Hydrogen atoms are omitted for clarity. Selected bond lengths/A and angles/deg: Ni(1)-O(2) 1.985(3), Ni(1)-O(1) 1.993(3), Ni(1)-N(7) 2.058(3), Ni(1)-N(8) 2.066(3), Ni(1)-O(3) 2.155(3), $\mathrm{Ni}(1)-\mathrm{O}(5) \quad 2.161(3), \quad \mathrm{Ni}(2)-\mathrm{O}(1) \quad 2.018(2), \quad \mathrm{Ni}(2)-\mathrm{O}(2) \quad 2.034(3), \quad \mathrm{Ni}(2)-\mathrm{N}(4) \quad 2.054(3)$, $\mathrm{Ni}(2)-\mathrm{O}(4)$ 2.062(3), Ni(2)-N(1) 2.079(3), Ni(2)-O(6) 2.102(3), O(2)-Ni(1)-O(1) 92.41(10), $\mathrm{O}(2)-\mathrm{Ni}(1)-(7) \quad$ 174.96(12), $\quad \mathrm{O}(1)-\mathrm{Ni}(1)-\mathrm{N}(7) \quad 88.96(12), \quad \mathrm{O}(2)-\mathrm{Ni}(1)-\mathrm{N}(8) \quad 89.84(12)$, $\mathrm{O}(1)-\mathrm{Ni}(1)-\mathrm{N}(8) \quad$ 173.80(12), $\quad \mathrm{N}(7)-\mathrm{Ni}(1)-\mathrm{N}(8) \quad 89.29(13), \quad \mathrm{O}(2)-\mathrm{Ni}(1)-\mathrm{O}(3) \quad 81.73(10)$, $\mathrm{O}(1)-\mathrm{Ni}(1)-\mathrm{O}(3) \quad 86.03(10), \quad \mathrm{N}(7)-\mathrm{Ni}(1)-\mathrm{O}(3) \quad 93.53(12), \quad \mathrm{N}(8)-\mathrm{Ni}(1)-\mathrm{O}(3) \quad$ 100.01(12), $\mathrm{O}(2)-\mathrm{Ni}(1)-\mathrm{O}(5) \quad 85.81(10), \quad \mathrm{O}(1)-\mathrm{Ni}(1)-\mathrm{O}(5) \quad 81.14(10), \quad \mathrm{N}(7)-\mathrm{Ni}(1)-\mathrm{O}(5) \quad 99.20(12)$, $\mathrm{N}(8)-\mathrm{Ni}(1)-\mathrm{O}(5) \quad 93.28(12), \quad \mathrm{O}(3)-\mathrm{Ni}(1)-\mathrm{O}(5) \quad 161.70(10), \quad \mathrm{O}(1)-\mathrm{Ni}(2)-\mathrm{O}(2) \quad 90.25(10)$, $\mathrm{O}(1)-\mathrm{Ni}(2)-\mathrm{N}(4) \quad$ 172.01(11), $\quad \mathrm{O}(2)-\mathrm{Ni}(2)-\mathrm{N}(4) \quad 82.22(11), \quad \mathrm{O}(1)-\mathrm{Ni}(2)-\mathrm{O}(4) \quad 85.80(10)$, $\mathrm{O}(2)-\mathrm{Ni}(2)-\mathrm{O}(4) \quad 87.59(10), \quad \mathrm{N}(4)-\mathrm{Ni}(2)-\mathrm{O}(4) \quad 91.17(11), \quad \mathrm{O}(1)-\mathrm{Ni}(2)-\mathrm{N}(1) \quad 83.03(11)$, $\mathrm{O}(2)-\mathrm{Ni}(2)-\mathrm{N}(1) \quad 172.69(11), \quad \mathrm{N}(4)-\mathrm{Ni}(2)-\mathrm{N}(1) \quad 104.62(12), \quad \mathrm{O}(4)-\mathrm{Ni}(2)-\mathrm{N}(1) \quad 94.75(11)$, $\mathrm{O}(1)-\mathrm{Ni}(2)-\mathrm{O}(6) \quad 86.68(10), \quad \mathrm{O}(2)-\mathrm{Ni}(2)-\mathrm{O}(6) \quad 85.21(10), \quad \mathrm{N}(4)-\mathrm{Ni}(2)-\mathrm{O}(6) \quad 95.32(11)$, $\mathrm{O}(4)-\mathrm{Ni}(2)-\mathrm{O}(6)$ 169.56(10), N(1)-Ni(2)-O(6) 91.51(11). 


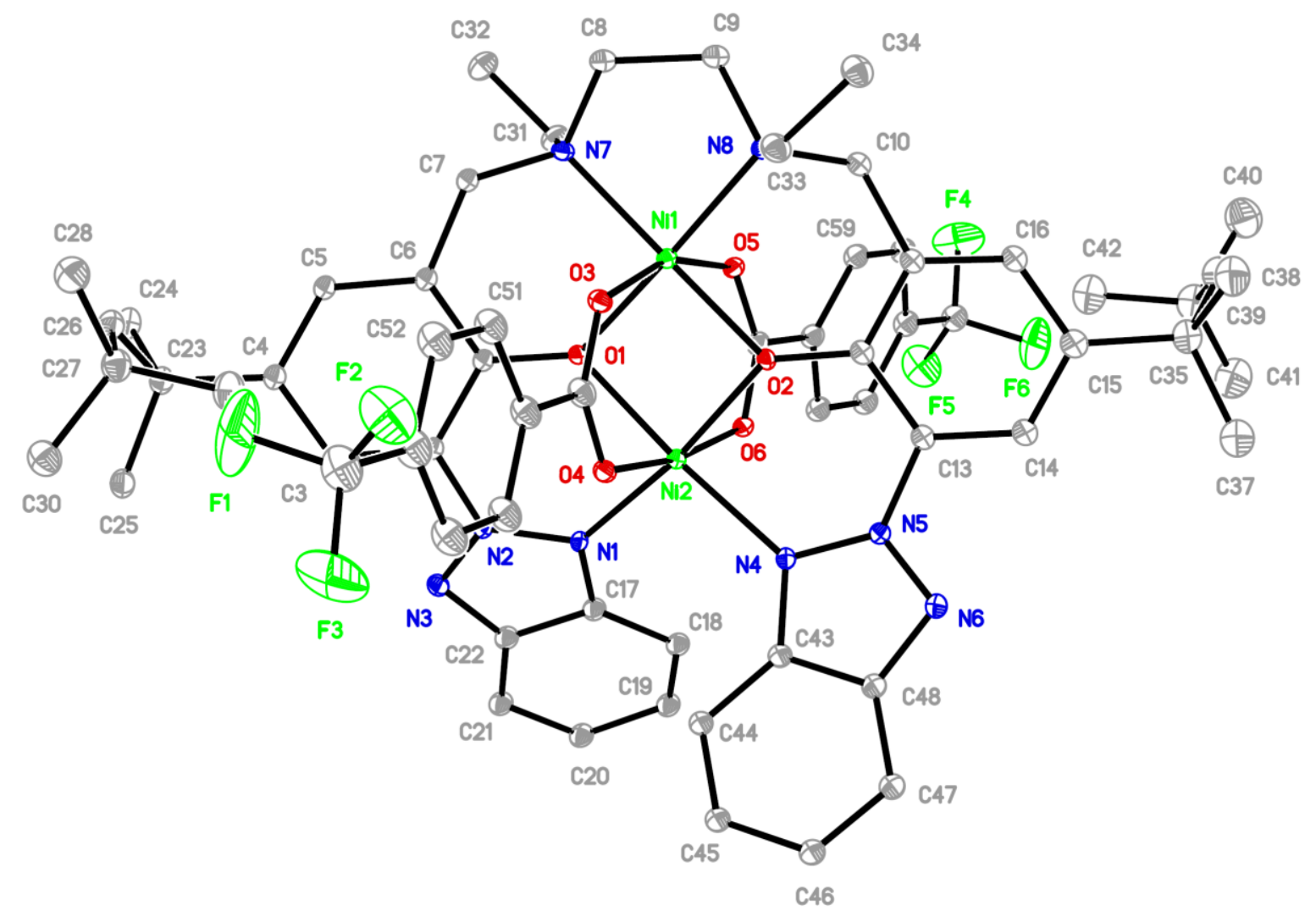

Figure S3. ORTEP drawing of complex 3 with probability ellipsoids drawn at level $20 \%$. Hydrogen atoms are omitted for clarity. Selected bond lengths/A and angles/deg: Ni(1)-O(1) 1.989(3), Ni(1)-O(3) 2.129(4), Ni(1)-O(2) 1.992(3), Ni(1)-O(5) 2.142(4), Ni(1)-N(7) 2.083(4), $\mathrm{Ni}(1)-\mathrm{N}(8) \quad 2.074(4), \quad \mathrm{Ni}(2)-\mathrm{O}(1) \quad 2.044(3), \quad \mathrm{Ni}(2)-\mathrm{O}(2) \quad 2.025(3), \quad \mathrm{Ni}(2)-\mathrm{O}(6) \quad 2.034(4)$, $\mathrm{Ni}(2)-\mathrm{O}(4)$ 2.055(4), Ni(2)-N(1) 2.048(4), Ni(2)-N(4) 2.091(4), O(1)-Ni(1)-O(3) 85.53(13), $\mathrm{O}(1)-\mathrm{Ni}(1)-\mathrm{O}(2) \quad 94.41(13), \quad \mathrm{O}(1)-\mathrm{Ni}(1)-\mathrm{O}(5) \quad 82.61(14), \quad \mathrm{O}(1)-\mathrm{Ni}(1)-\mathrm{N}(7) \quad 88.67(15)$, $\mathrm{O}(1)-\mathrm{Ni}(1)-\mathrm{N}(8) \quad$ 175.01(16), $\quad \mathrm{O}(3)-\mathrm{Ni}(1)-\mathrm{O}(5) \quad 161.36(14), \quad \mathrm{O}(2)-\mathrm{Ni}(1)-\mathrm{O}(3) \quad 81.16(14)$, $\mathrm{O}(2)-\mathrm{Ni}(1)-\mathrm{O}(5) \quad 85.46(14), \quad \mathrm{O}(2)-\mathrm{Ni}(1)-\mathrm{N}(7) \quad$ 173.62(15), $\quad \mathrm{O}(2)-\mathrm{Ni}(1)-\mathrm{N}(8) \quad 88.56(15)$, $\mathrm{N}(7)-\mathrm{Ni}(1)-\mathrm{O}(3) \quad 93.52(15) ， \quad \mathrm{~N}(7)-\mathrm{Ni}(1)-\mathrm{O}(5) \quad$ 100.50(15)， N(8)-Ni(1)-Ni(2) $\quad 135.28(12)$, $\mathrm{N}(8)-\mathrm{Ni}(1)-\mathrm{O}(3) \quad 98.90(15), \quad \mathrm{N}(8)-\mathrm{Ni}(1)-\mathrm{O}(5) \quad 93.65(15), \quad \mathrm{N}(8)-\mathrm{Ni}(1)-\mathrm{N}(7) \quad 88.78(17)$, $\mathrm{O}(1)-\mathrm{Ni}(2)-\mathrm{O}(4) \quad 87.06(14), \quad \mathrm{O}(1)-\mathrm{Ni}(2)-\mathrm{N}(1) \quad 81.00(14), \quad \mathrm{O}(1)-\mathrm{Ni}(2)-\mathrm{N}(4) \quad$ 174.70(15), $\mathrm{O}(2)-\mathrm{Ni}(2)-\mathrm{O}(1) \quad 91.77(13), \quad \mathrm{O}(2)-\mathrm{Ni}(2)-\mathrm{O}(6) \quad 87.28(14), \quad \mathrm{O}(2)-\mathrm{Ni}(2)-\mathrm{O}(4) \quad 86.15(14)$, $\mathrm{O}(2)-\mathrm{Ni}(2)-\mathrm{N}(1) \quad$ 172.61(15), $\quad \mathrm{O}(2)-\mathrm{Ni}(2)-\mathrm{N}(4) \quad 83.03(15), \quad \mathrm{O}(6)-\mathrm{Ni}(2)-\mathrm{Ni}(1) \quad 85.66(10)$, $\mathrm{O}(6)-\mathrm{Ni}(2)-\mathrm{O}(1) \quad 87.50(13), \quad \mathrm{O}(6)-\mathrm{Ni}(2)-\mathrm{O}(4) \quad 171.34(14), \quad \mathrm{O}(6)-\mathrm{Ni}(2)-\mathrm{N}(1) \quad 90.82(15)$, $\mathrm{O}(6)-\mathrm{Ni}(2)-\mathrm{N}(4) \quad 93.24(15), \quad \mathrm{O}(4)-\mathrm{Ni}(2)-\mathrm{Ni}(1) \quad 85.71(10), \quad \mathrm{O}(4)-\mathrm{Ni}(2)-\mathrm{N}(4) \quad 91.56(15)$, $\mathrm{N}(1)-\mathrm{Ni}(2)-\mathrm{Ni}(1)$ 126.79(11), N(1)-Ni(2)-O(4) 94.98(15), N(1)-Ni(2)-N(4) 104.23(16). 


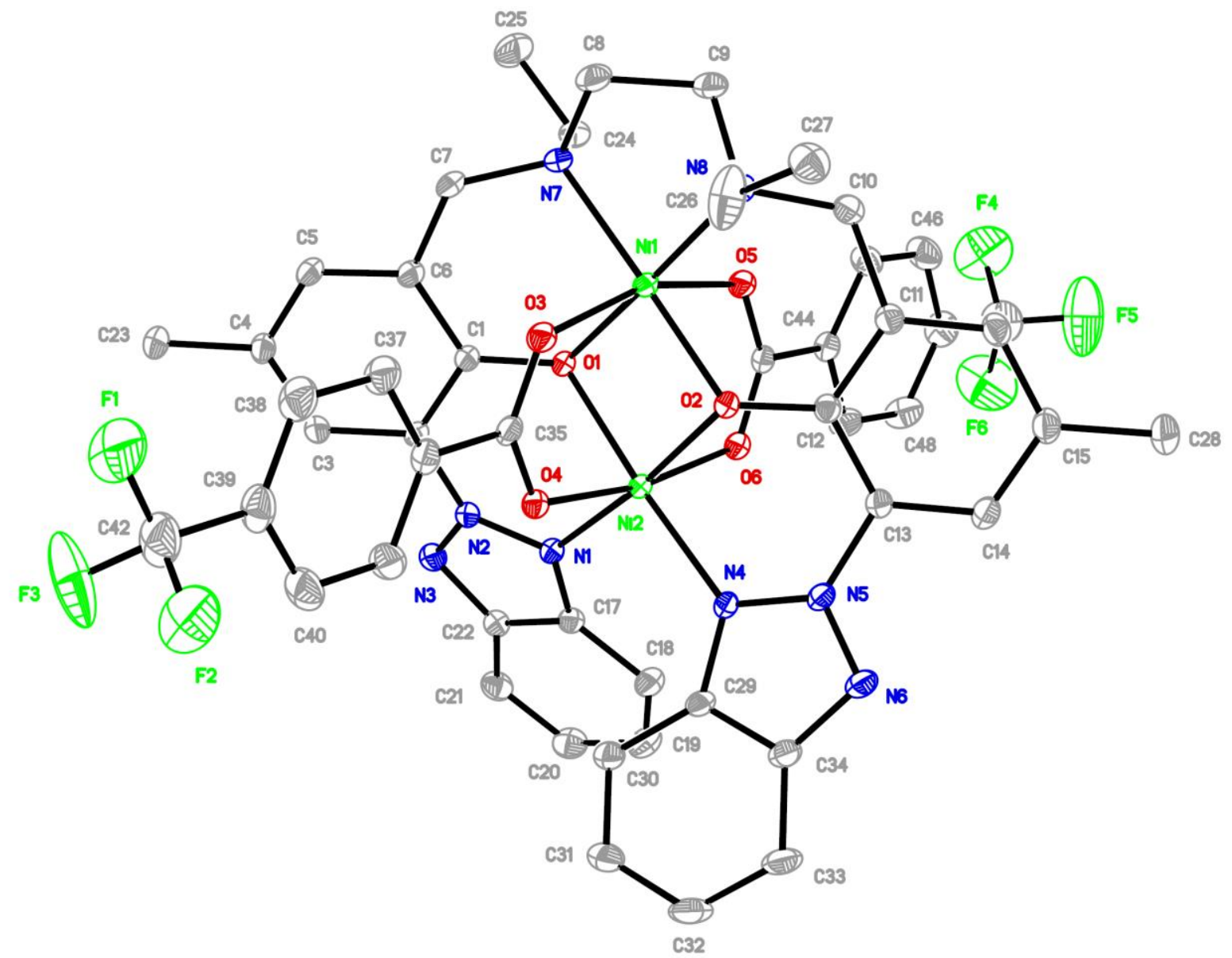

Figure S4. ORTEP drawing of complex 4 with probability ellipsoids drawn at level $20 \%$. Hydrogen atoms are omitted for clarity. Selected bond lengths/A and angles/deg: Ni(1)-O(2) 1.9842(17), Ni(1)-O(1) 1.9916(17), Ni(1)-N(8) 2.077(2), Ni(1)-N(7) 2.079(2), Ni(1)-O(3) 2.1304(18), Ni(1)-O(5) 2.1419(18), Ni(2)-O(2) 2.0310(17), Ni(2)-O(6) 2.0335(17), Ni(2)-O(1) 2.0359(17), Ni(2)-O(4) 2.0430(18), Ni(2)-N(4) 2.082(2), Ni(2)-N(1) 2.090(2), O(2)-Ni(1)-O(1) 94.21(7), O(2)-Ni(1)-N(8) 89.09(8), O(1)-Ni(1)-N(8) 174.19(8), O(2)-Ni(1)-N(7) 174.39(8), $\mathrm{O}(1)-\mathrm{Ni}(1)-\mathrm{N}(7) \quad 88.40(8), \quad \mathrm{N}(8)-\mathrm{Ni}(1)-\mathrm{N}(7) \quad 88.73(8), \quad \mathrm{O}(2)-\mathrm{Ni}(1)-\mathrm{O}(3) \quad 81.93(7)$, $\mathrm{O}(1)-\mathrm{Ni}(1)-\mathrm{O}(3) \quad 86.11(7), \quad \mathrm{N}(8)-\mathrm{Ni}(1)-\mathrm{O}(3) \quad 99.11(8), \quad \mathrm{N}(7)-\mathrm{Ni}(1)-\mathrm{O}(3) \quad 93.31(8)$, $\mathrm{O}(2)-\mathrm{Ni}(1)-\mathrm{O}(5) \quad 85.73(7), \quad \mathrm{O}(1)-\mathrm{Ni}(1)-\mathrm{O}(5) \quad 81.37(7), \quad \mathrm{N}(8)-\mathrm{Ni}(1)-\mathrm{O}(5) \quad 94.12(8)$, $\mathrm{N}(7)-\mathrm{Ni}(1)-\mathrm{O}(5) \quad 99.59(8), \quad \mathrm{O}(3)-\mathrm{Ni}(1)-\mathrm{O}(5) \quad 161.71(7), \quad \mathrm{O}(2)-\mathrm{Ni}(2)-\mathrm{O}(6) \quad 86.69(7)$, $\mathrm{O}(2)-\mathrm{Ni}(2)-\mathrm{O}(1) \quad 91.49(7), \quad \mathrm{O}(6)-\mathrm{Ni}(2)-\mathrm{O}(1) \quad 85.93(7), \quad \mathrm{O}(2)-\mathrm{Ni}(2)-\mathrm{O}(4) \quad 87.21(7)$, $\mathrm{O}(6)-\mathrm{Ni}(2)-\mathrm{O}(4) 170.44(7), \quad \mathrm{O}(1)-\mathrm{Ni}(2)-\mathrm{O}(4) \quad 86.88(7), \quad \mathrm{O}(2)-\mathrm{Ni}(2)-\mathrm{N}(4) \quad 81.44(7)$, $\mathrm{O}(6)-\mathrm{Ni}(2)-\mathrm{N}(4) \quad 96.24(8), \quad \mathrm{O}(1)-\mathrm{Ni}(2)-\mathrm{N}(4) \quad 172.45(8), \quad \mathrm{O}(4)-\mathrm{Ni}(2)-\mathrm{N}(4) \quad 90.12(8)$, $\mathrm{O}(2)-\mathrm{Ni}(2)-\mathrm{N}(1) \quad$ 173.79(7), $\quad \mathrm{O}(6)-\mathrm{Ni}(2)-\mathrm{N}(1) \quad 91.31(8), \quad \mathrm{O}(1)-\mathrm{Ni}(2)-\mathrm{N}(1) \quad 82.51(7)$, $\mathrm{O}(4)-\mathrm{Ni}(2)-\mathrm{N}(1)$ 94.00(8), N(4)-Ni(2)-N(1) 104.63(8). 


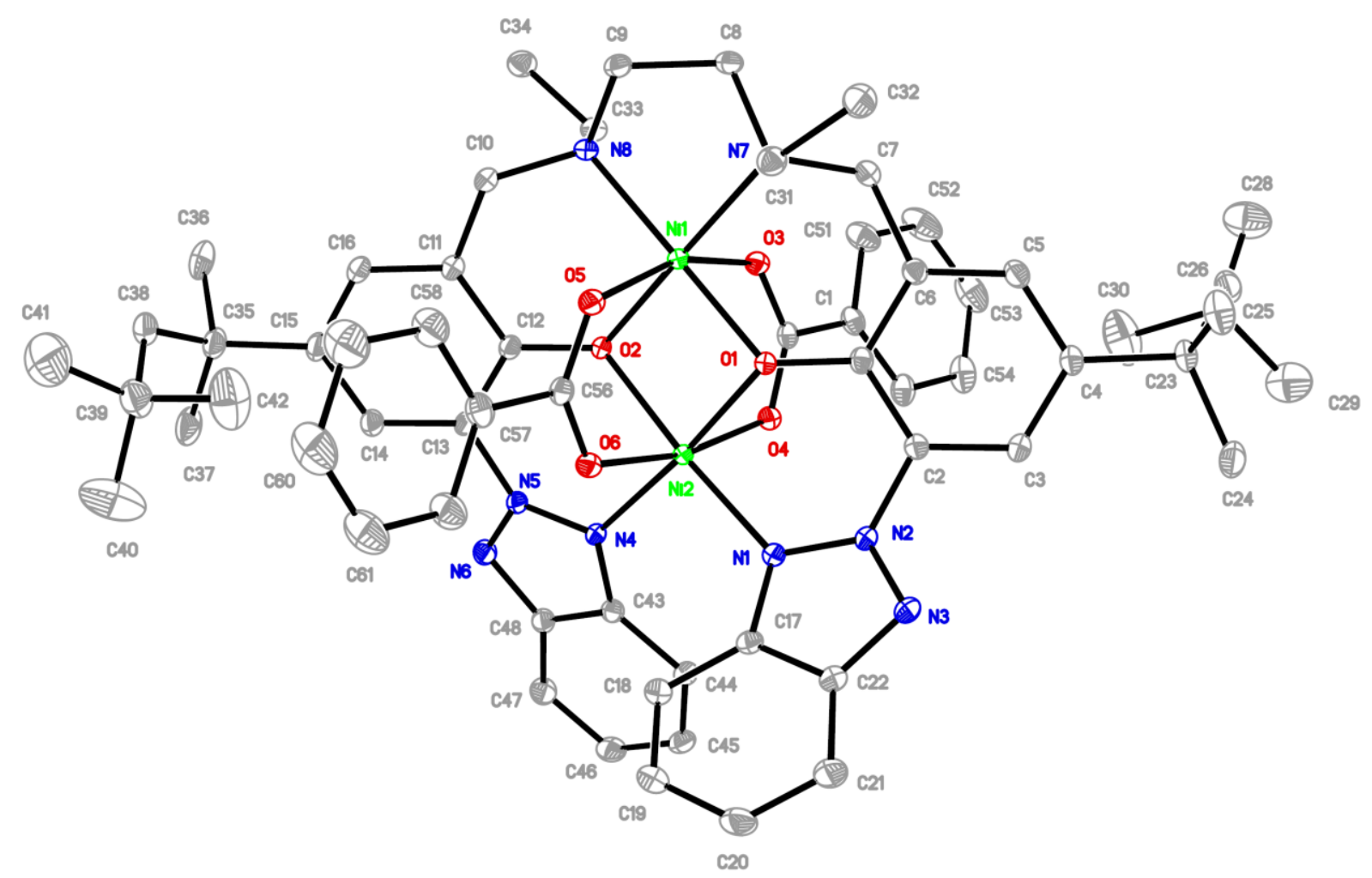

Figure S5. ORTEP drawing of complex 5 with probability ellipsoids drawn at level $20 \%$. Hydrogen atoms are omitted for clarity. Selected bond lengths/A and angles/deg: Ni(1)-O(1) 1.9840(12), Ni(1)-O(2) 1.9980(13), Ni(1)-N(8) 2.0651(15), Ni(1)-N(7) 2.0727(16), Ni(1)-O(3) 2.1042(13), Ni(1)-O(5) 2.1071(13), Ni(2)-O(2) 2.0182(12), Ni(2)-O(1) 2.0271(13), Ni(2)-O(6) 2.0380(13), $\mathrm{Ni}(2)-\mathrm{O}(4) \quad 2.0679(13), \quad \mathrm{Ni}(2)-\mathrm{N}(1) \quad 2.0761(15), \quad \mathrm{Ni}(2)-\mathrm{N}(4) \quad 2.1128(16)$, $\mathrm{O}(1)-\mathrm{Ni}(1)-\mathrm{O}(2) \quad 93.44(5), \quad \mathrm{O}(1)-\mathrm{Ni}(1)-\mathrm{N}(8) \quad$ 174.24(6), $\quad \mathrm{O}(2)-\mathrm{Ni}(1)-\mathrm{N}(8) \quad 89.54(6)$, $\mathrm{O}(1)-\mathrm{Ni}(1)-\mathrm{N}(7) \quad 88.30(6), \quad \mathrm{O}(2)-\mathrm{Ni}(1)-\mathrm{N}(7) \quad 172.88(6), \quad \mathrm{N}(8)-\mathrm{Ni}(1)-\mathrm{N}(7) \quad 89.34(6)$, $\mathrm{O}(1)-\mathrm{Ni}(1)-\mathrm{O}(3) \quad 86.34(5), \quad \mathrm{O}(2)-\mathrm{Ni}(1)-\mathrm{O}(3) \quad 79.79(5), \quad \mathrm{N}(8)-\mathrm{Ni}(1)-\mathrm{O}(3) \quad 99.05(6)$, $\mathrm{N}(7)-\mathrm{Ni}(1)-\mathrm{O}(3) \quad 93.45(6), \quad \mathrm{O}(1)-\mathrm{Ni}(1)-\mathrm{O}(5) \quad 82.16(5), \quad \mathrm{O}(2)-\mathrm{Ni}(1)-\mathrm{O}(5) \quad 87.35(5)$, $\mathrm{N}(8)-\mathrm{Ni}(1)-\mathrm{O}(5) \quad 93.07(6), \quad \mathrm{N}(7)-\mathrm{Ni}(1)-\mathrm{O}(5) \quad 99.73(6), \quad \mathrm{O}(3)-\mathrm{Ni}(1)-\mathrm{O}(5) \quad$ 162.19(5), $\mathrm{O}(2)-\mathrm{Ni}(2)-\mathrm{O}(1) \quad 91.56(5), \quad \mathrm{O}(2)-\mathrm{Ni}(2)-\mathrm{O}(6) \quad 85.89(5), \quad \mathrm{O}(1)-\mathrm{Ni}(2)-\mathrm{O}(6) \quad 87.79(5)$, $\mathrm{O}(2)-\mathrm{Ni}(2)-\mathrm{O}(4) \quad 85.90(5), \quad \mathrm{O}(1)-\mathrm{Ni}(2)-\mathrm{O}(4) \quad 85.46(5), \quad \mathrm{O}(6)-\mathrm{Ni}(2)-\mathrm{O}(4) \quad$ 169.22(5), $\mathrm{O}(2)-\mathrm{Ni}(2)-\mathrm{N}(1) \quad 170.52(6), \quad \mathrm{O}(1)-\mathrm{Ni}(2)-\mathrm{N}(1) \quad 80.17(6), \quad \mathrm{O}(6)-\mathrm{Ni}(2)-\mathrm{N}(1) \quad 89.14(6)$, $\mathrm{O}(4)-\mathrm{Ni}(2)-\mathrm{N}(1) \quad 97.96(6), \quad \mathrm{O}(2)-\mathrm{Ni}(2)-\mathrm{N}(4) \quad 83.31(5), \quad \mathrm{O}(1)-\mathrm{Ni}(2)-\mathrm{N}(4) \quad$ 174.82(5), $\mathrm{O}(6)-\mathrm{Ni}(2)-\mathrm{N}(4)$ 92.49(6), O(4)-Ni(2)-N(4) 93.47(6), N(1)-Ni(2)-N(4) 105.00(6). 


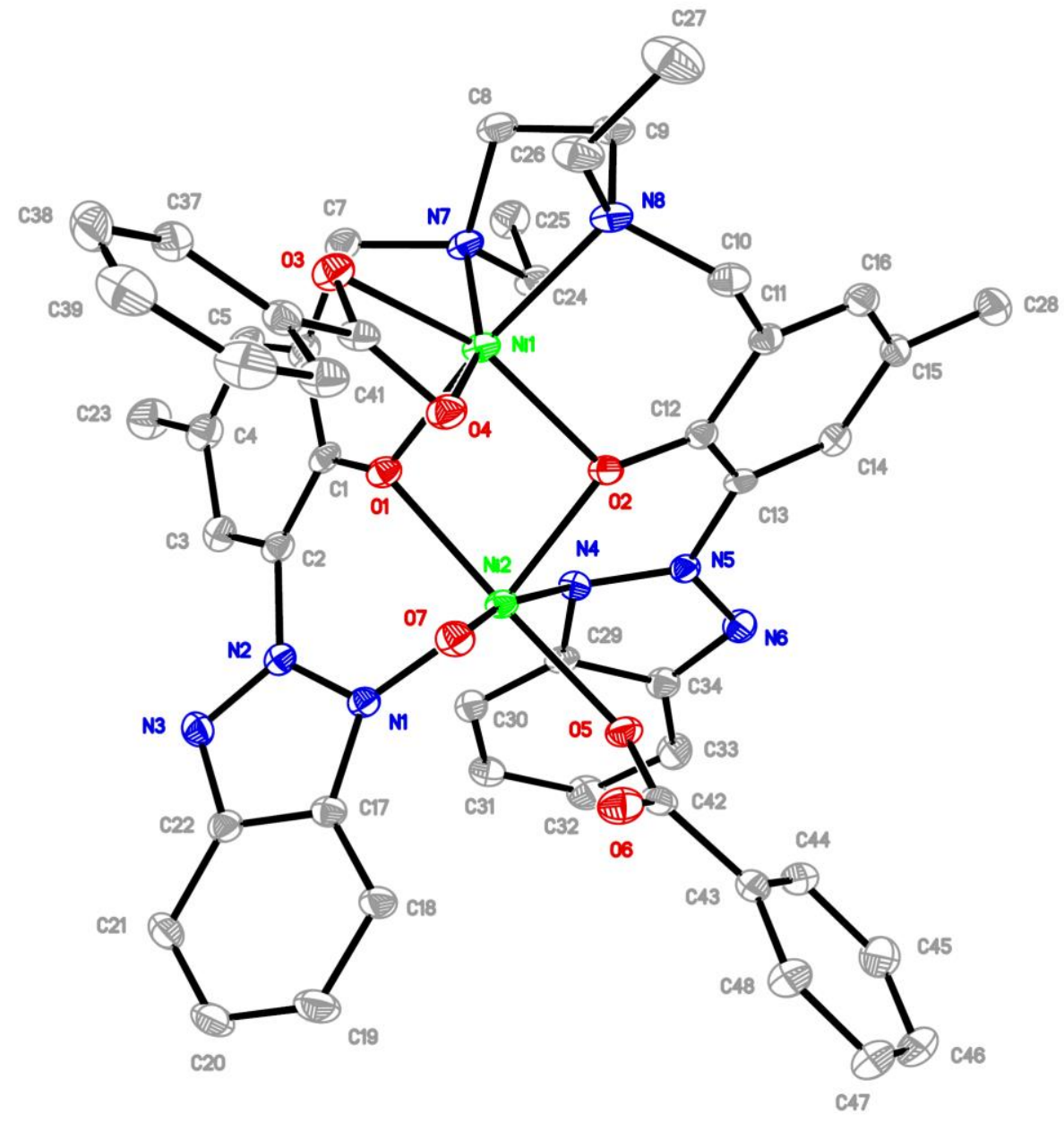

Figure S6. ORTEP drawing of complex 6 with probability ellipsoids drawn at level $30 \%$. Hydrogen atoms are omitted for clarity. Selected bond lengths/ $\AA$ and angles/deg: Ni(1)-O(2) 2.045(3), Ni(1)-O(1) 2.066(3), Ni(1)-N(7) 2.075(3), Ni(1)-O(3) 2.127(3), Ni(1)-O(4) 2.160(3), $\mathrm{Ni}(1)-\mathrm{N}(8) \quad 2.171(3), \quad \mathrm{Ni}(2)-\mathrm{O}(2) \quad 2.025(3), \quad \mathrm{Ni}(2)-\mathrm{O}(7) \quad 2.030(3), \quad \mathrm{Ni}(2)-\mathrm{O}(5) \quad 2.054(3)$, $\mathrm{Ni}(2)-\mathrm{O}(1)$ 2.062(3), Ni(2)-N(1) 2.060(3), Ni(2)-N(4) 2.164(3), O(2)-Ni(1)-O(1) 79.63(10), $\mathrm{O}(2)-\mathrm{Ni}(1)-\mathrm{N}(7) \quad 108.57(12), \quad \mathrm{O}(1)-\mathrm{Ni}(1)-\mathrm{N}(7) \quad 88.95(12), \quad \mathrm{O}(2)-\mathrm{Ni}(1)-\mathrm{O}(3) \quad$ 154.48(10), $\mathrm{O}(1)-\mathrm{Ni}(1)-\mathrm{O}(3) \quad 94.20(11), \quad \mathrm{N}(7)-\mathrm{Ni}(1)-\mathrm{O}(3) \quad 95.94(11), \quad \mathrm{O}(2)-\mathrm{Ni}(1)-\mathrm{O}(4) \quad 94.04(10)$, $\mathrm{O}(1)-\mathrm{Ni}(1)-\mathrm{O}(4) \quad 95.65(10), \quad \mathrm{N}(7)-\mathrm{Ni}(1)-\mathrm{O}(4) \quad$ 157.39(12), $\quad \mathrm{O}(3)-\mathrm{Ni}(1)-\mathrm{O}(4) \quad 61.70(10)$, $\mathrm{O}(2)-\mathrm{Ni}(1)-\mathrm{N}(8) \quad 92.65(12), \quad \mathrm{O}(1)-\mathrm{Ni}(1)-\mathrm{N}(8) \quad$ 169.04(12), $\quad \mathrm{N}(7)-\mathrm{Ni}(1)-\mathrm{N}(8) \quad 86.14(13)$, $\mathrm{O}(3)-\mathrm{Ni}(1)-\mathrm{N}(8) \quad 6.05(12), \quad \mathrm{O}(4)-\mathrm{Ni}(1)-\mathrm{N}(8) \quad 2.63(11), \quad \mathrm{O}(2)-\mathrm{Ni}(1)-\mathrm{C}(35) \quad 24.92(12)$, $\mathrm{O}(1)-\mathrm{Ni}(1)-\mathrm{C}(35) \quad 6.55(12), \quad \mathrm{N}(7)-\mathrm{Ni}(1)-\mathrm{C}(35) \quad$ 126.39(13), $\quad \mathrm{O}(3)-\mathrm{Ni}(1)-\mathrm{C}(35) \quad 30.59(11)$, $\mathrm{O}(4)-\mathrm{Ni}(1)-\mathrm{C}(35) \quad 31.13(11), \quad \mathrm{N}(8)-\mathrm{Ni}(1)-\mathrm{C}(35) \quad 94.25(13), \quad \mathrm{O}(2)-\mathrm{Ni}(2)-\mathrm{O}(7) \quad 93.01(11)$, $\mathrm{O}(2)-\mathrm{Ni}(2)-\mathrm{O}(5) \quad 97.45(11), \quad \mathrm{O}(7)-\mathrm{Ni}(2)-\mathrm{O}(5) \quad 90.21(11), \quad \mathrm{O}(2)-\mathrm{Ni}(2)-\mathrm{O}(1) \quad 80.20(11)$, $\mathrm{O}(7)-\mathrm{Ni}(2)-\mathrm{O}(1) \quad 88.47(10), \quad \mathrm{O}(5)-\mathrm{Ni}(2)-\mathrm{O}(1) \quad 177.23(11), \quad \mathrm{O}(2)-\mathrm{Ni}(2)-\mathrm{N}(1) \quad$ 164.01(12), $\mathrm{O}(7)-\mathrm{Ni}(2)-\mathrm{N}(1) \quad 85.89(12), \quad \mathrm{O}(5)-\mathrm{Ni}(2)-\mathrm{N}(1) \quad 98.50(12), \quad \mathrm{O}(1)-\mathrm{Ni}(2)-\mathrm{N}(1) \quad 83.83(11)$, $\mathrm{O}(2)-\mathrm{Ni}(2)-\mathrm{N}(4) \quad 85.70(11), \quad \mathrm{O}(7)-\mathrm{Ni}(2)-\mathrm{N}(4) \quad$ 169.81(11), $\quad \mathrm{O}(5)-\mathrm{Ni}(2)-\mathrm{N}(4) \quad 79.95(11)$, $\mathrm{O}(1)-\mathrm{Ni}(2)-\mathrm{N}(4)$ 101.25(11), N(1)-Ni(2)-N(4) 98.11(12). 


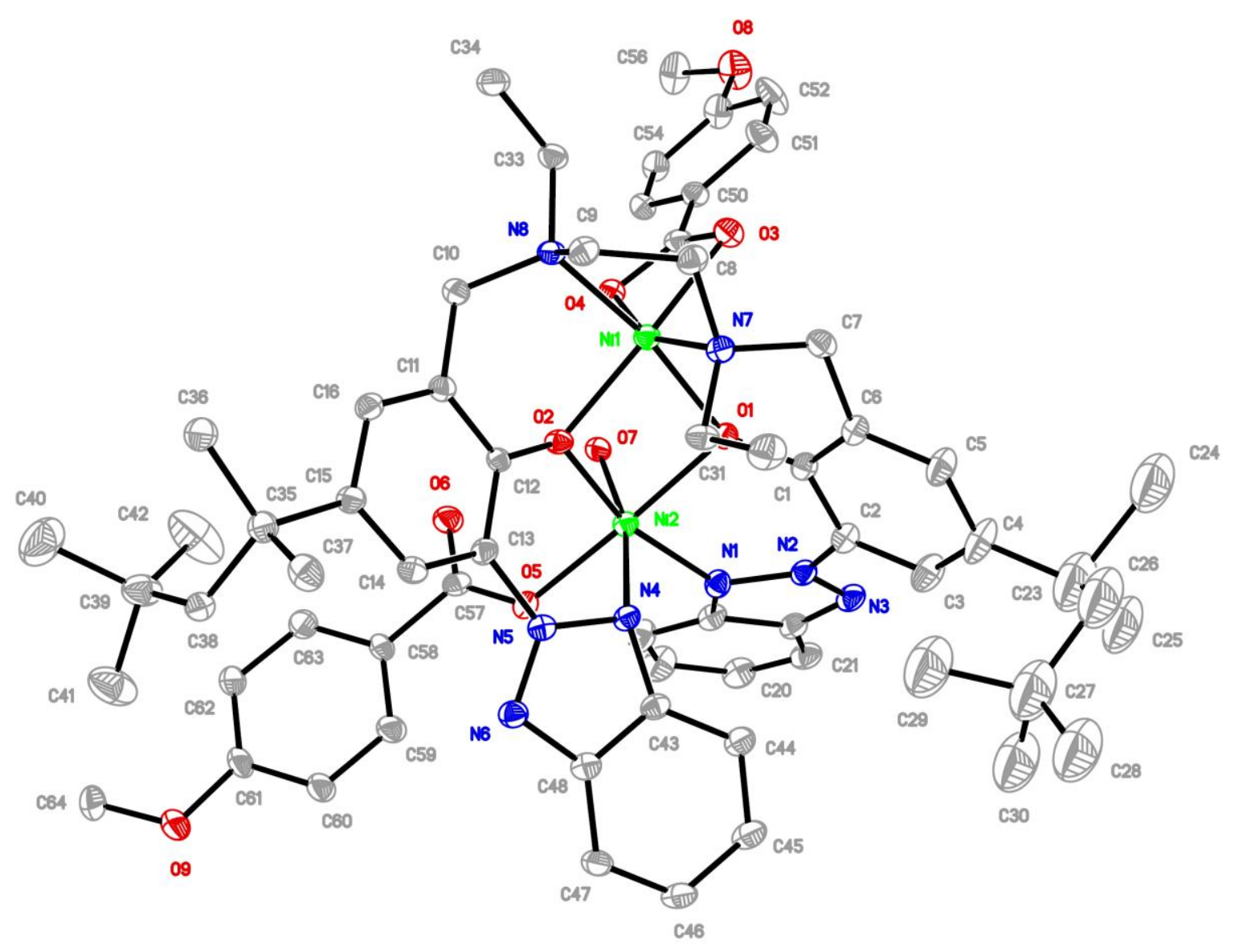

Figure S7. ORTEP drawing of complex 7 with probability ellipsoids drawn at level $30 \%$. Hydrogen atoms are omitted for clarity. Selected bond lengths/A and angles/deg: Ni(1)-O(2) 2.018(4), Ni(1)-O(1) 2.054(4), Ni(1)-N(7) 2.084(5), Ni(1)-O(4) 2.095(4), Ni(1)-N(8) 2.117(5), $\mathrm{Ni}(1)-\mathrm{O}(3)$ 2.187(4), $\mathrm{Ni}(1)-\mathrm{C}(49)$ 2.471(6), $\mathrm{Ni}(2)-\mathrm{O}(2)$ 2.006(4), $\mathrm{Ni}(2)-\mathrm{O}(5)$ 2.025(4), $\mathrm{Ni}(2)-\mathrm{N}(1) \quad 2.045(5), \quad \mathrm{Ni}(2)-\mathrm{O}(1) \quad 2.061(4), \quad \mathrm{Ni}(2)-\mathrm{O}(7) \quad 2.064(4), \quad \mathrm{Ni}(2)-\mathrm{N}(4) \quad 2.215(4)$, $\mathrm{O}(2)-\mathrm{Ni}(1)-\mathrm{O}(1) \quad 79.12(16), \quad \mathrm{O}(2)-\mathrm{Ni}(1)-\mathrm{N}(7) \quad$ 105.94(18), $\quad \mathrm{O}(1)-\mathrm{Ni}(1)-\mathrm{N}(7) \quad 89.51(17)$, $\mathrm{O}(2)-\mathrm{Ni}(1)-\mathrm{O}(4) \quad 93.95(16), \quad \mathrm{O}(1)-\mathrm{Ni}(1)-\mathrm{O}(4) \quad 91.74(15), \quad \mathrm{N}(7)-\mathrm{Ni}(1)-\mathrm{O}(4) \quad$ 159.94(18), $\mathrm{O}(2)-\mathrm{Ni}(1)-\mathrm{N}(8) \quad 91.67(17), \quad \mathrm{O}(1)-\mathrm{Ni}(1)-\mathrm{N}(8) \quad$ 168.66(17), $\quad \mathrm{N}(7)-\mathrm{Ni}(1)-\mathrm{N}(8) \quad$ 86.66(19), $\mathrm{O}(4)-\mathrm{Ni}(1)-\mathrm{N}(8) \quad 95.51(17), \quad \mathrm{O}(2)-\mathrm{Ni}(1)-\mathrm{O}(3) \quad$ 154.11(16), $\quad \mathrm{O}(1)-\mathrm{Ni}(1)-\mathrm{O}(3) \quad 91.88(16)$, $\mathrm{N}(7)-\mathrm{Ni}(1)-\mathrm{O}(3) \quad 98.13(18), \quad \mathrm{O}(4)-\mathrm{Ni}(1)-\mathrm{O}(3) \quad 61.83(15), \quad \mathrm{N}(8)-\mathrm{Ni}(1)-\mathrm{O}(3) \quad 99.22(17)$, $\mathrm{O}(2)-\mathrm{Ni}(2)-\mathrm{O}(5) \quad 98.49(16), \quad \mathrm{O}(2)-\mathrm{Ni}(2)-\mathrm{N}(1) \quad$ 164.68(19), $\quad \mathrm{O}(5)-\mathrm{Ni}(2)-\mathrm{N}(1) \quad$ 96.81(19), $\mathrm{O}(2)-\mathrm{Ni}(2)-\mathrm{O}(1) \quad 79.21(16), \quad \mathrm{O}(5)-\mathrm{Ni}(2)-\mathrm{O}(1) \quad 177.51(16), \quad \mathrm{N}(1)-\mathrm{Ni}(2)-\mathrm{O}(1) \quad 85.50(18)$, $\mathrm{O}(2)-\mathrm{Ni}(2)-\mathrm{O}(7) \quad 92.99(16), \quad \mathrm{O}(5)-\mathrm{Ni}(2)-\mathrm{O}(7) \quad 90.08(16), \quad \mathrm{N}(1)-\mathrm{Ni}(2)-\mathrm{O}(7) \quad 87.70(18)$, $\mathrm{O}(1)-\mathrm{Ni}(2)-\mathrm{O}(7) \quad 89.10(16), \quad \mathrm{O}(2)-\mathrm{Ni}(2)-\mathrm{N}(4) \quad 83.52(16), \quad \mathrm{O}(5)-\mathrm{Ni}(2)-\mathrm{N}(4) \quad 79.32(17)$, $\mathrm{N}(1)-\mathrm{Ni}(2)-\mathrm{N}(4)$ 98.65(19), O(1)-Ni(2)-N(4) 101.27(17), O(7)-Ni(2)-N(4) 168.18(17). 


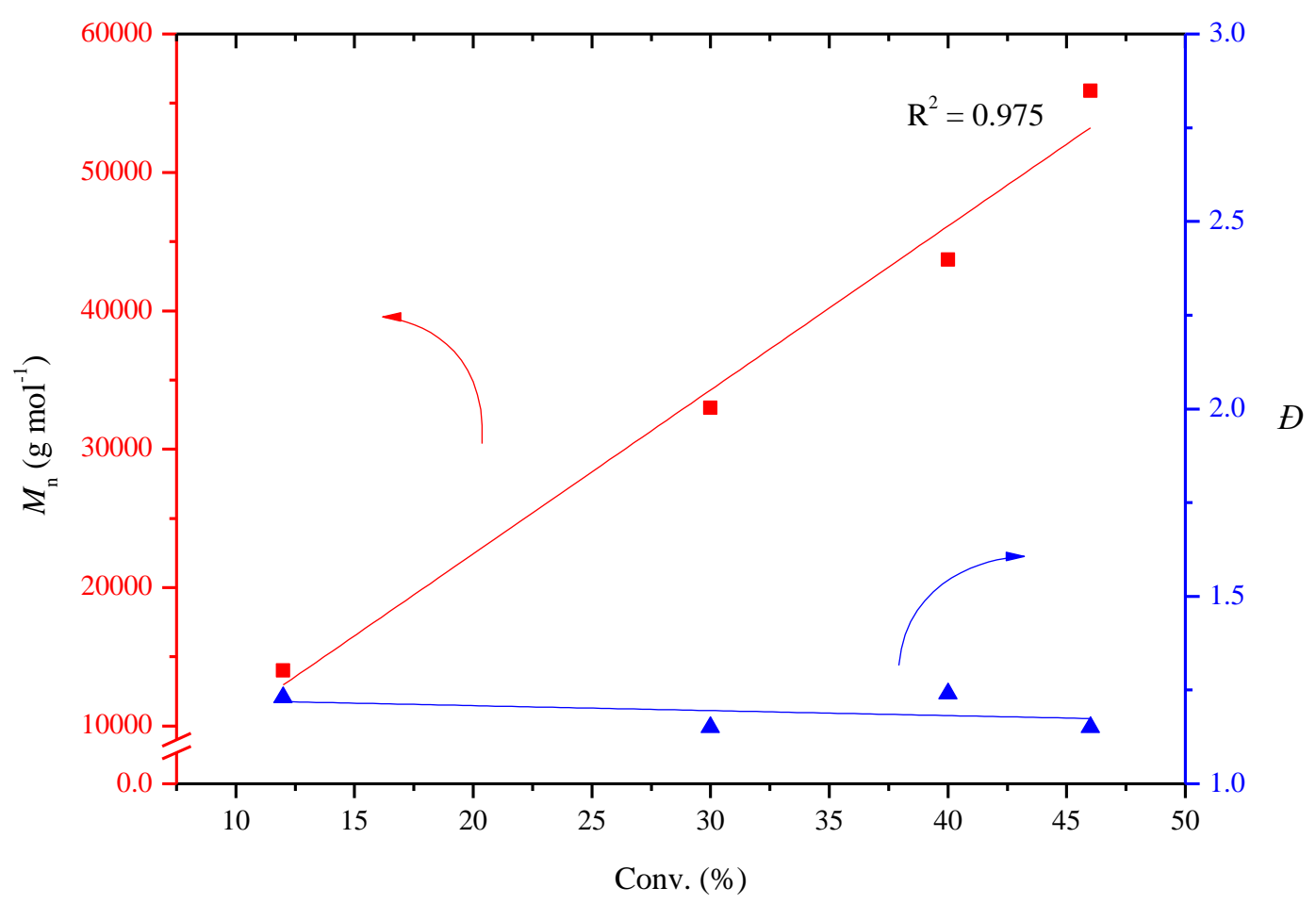

Figure S8. The plot of Mn ( $\bullet$ ) and PDI ( $\mathbf{\Delta})$ (determined from GPC analysis) versus CHO conversion for $\mathrm{CO}_{2}$-copolymerization of $\mathrm{CHO}$ using dinickel complex $\mathbf{1}$ as the catalyst $\left([\mathrm{CHO}]_{0} /[\mathbf{1}]_{0}=3200\right)$ at $140{ }^{\circ} \mathrm{C}$ and $300 \mathrm{psi} \mathrm{CO}$. 


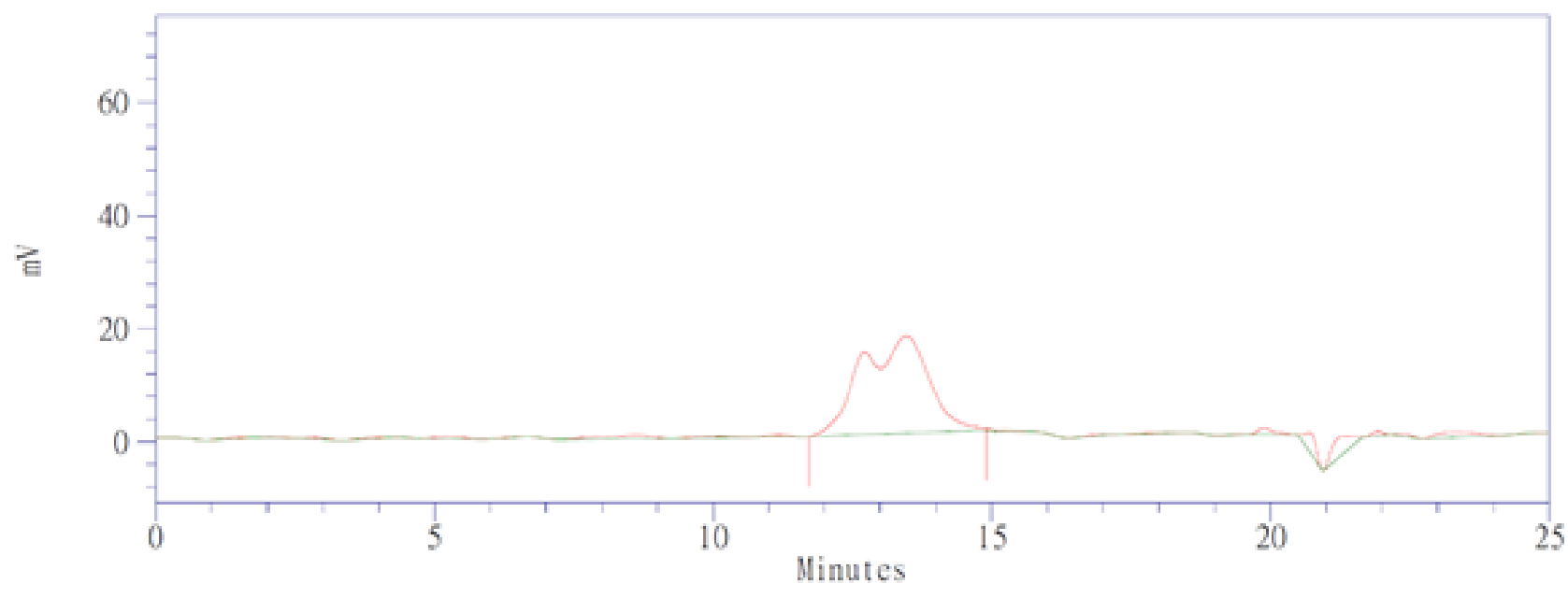

Figure S9. GPC traces for the generated PCHC with a bimodal molecular weight distribution catalyzed by dinickel complex 1 (Table 2, entry18). 


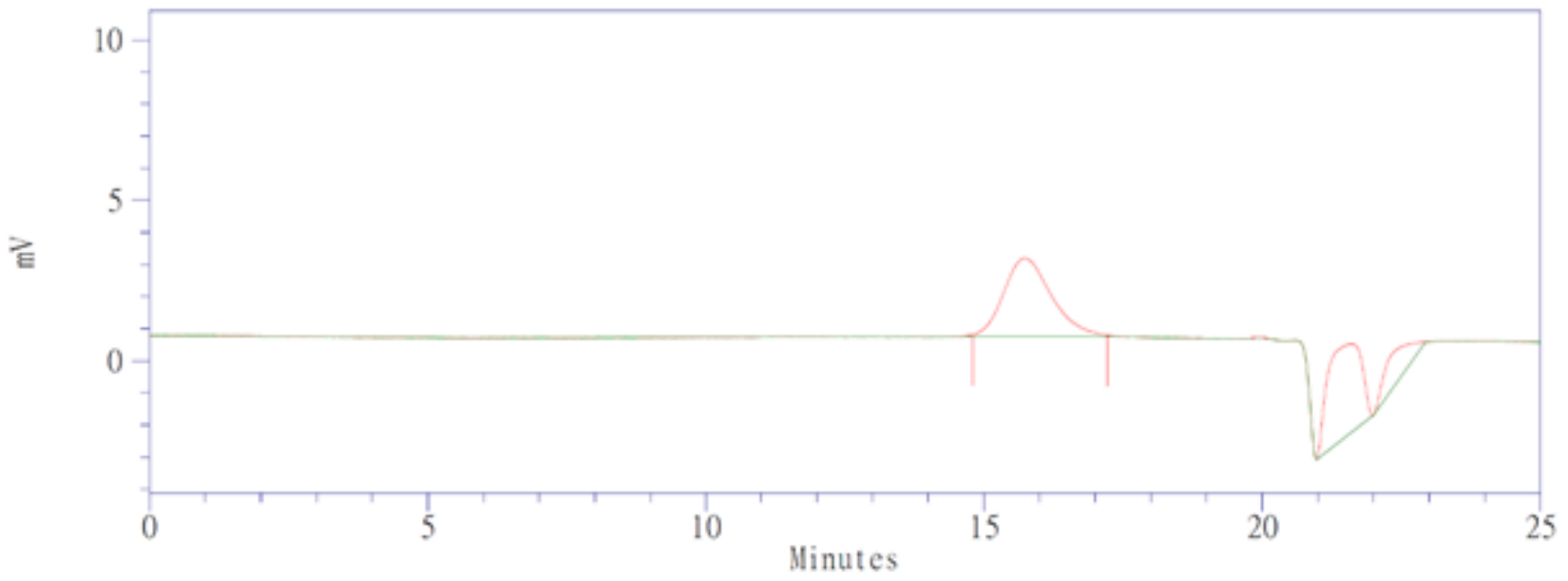

Figure S10. GPC traces for the obtained PCHC polyol with a unimodal molecular weight distribution catalyzed by dinickel complex 1 on the addition of $\mathrm{H}_{2} \mathrm{O}$ as the CTA (Table 3, entry 2). 

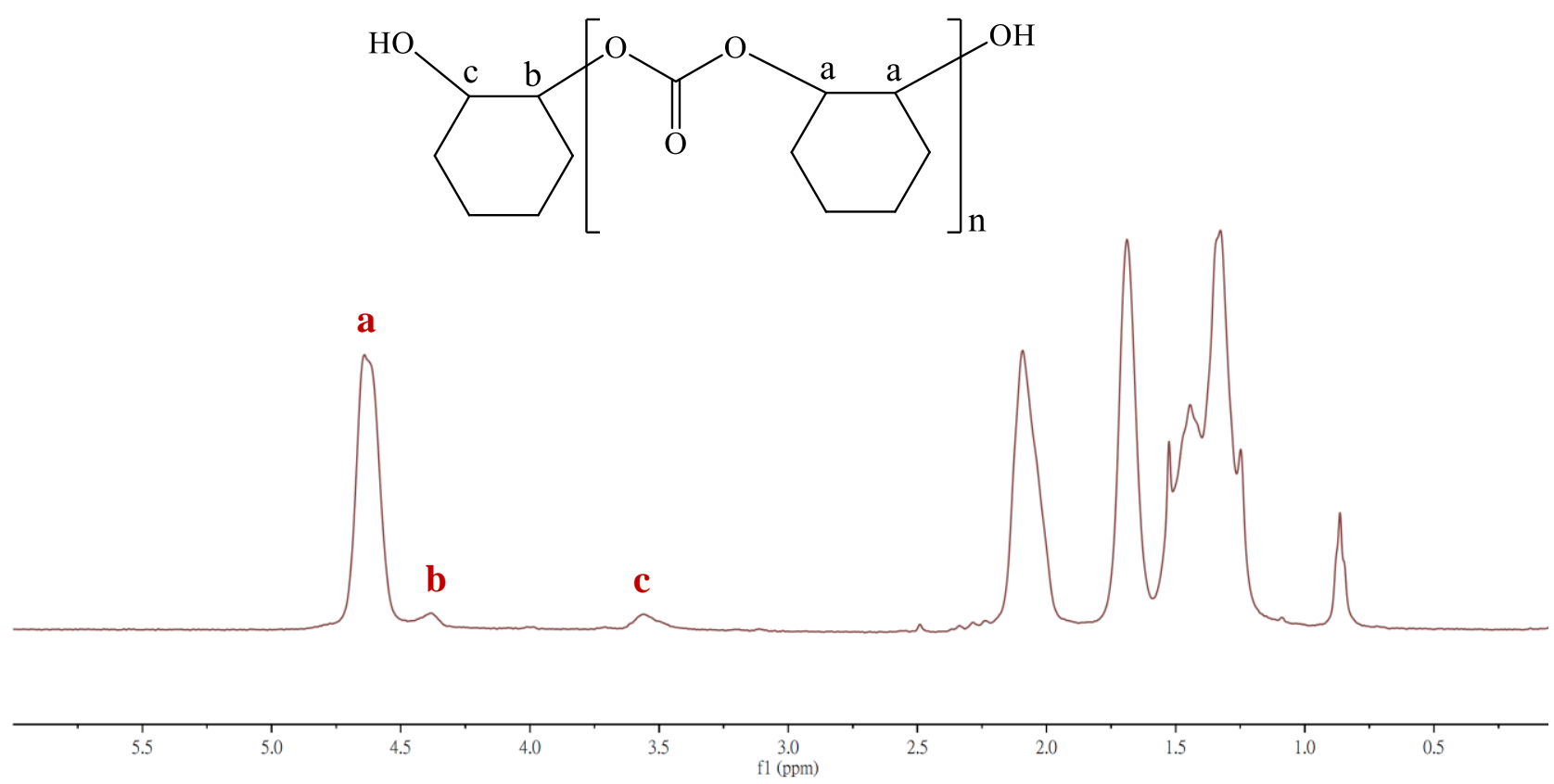

Figure S11. ${ }^{1} \mathrm{H}$ NMR spectrum of the purified copolymer generated by using dinickel complex 1 on the addition of $\mathrm{H}_{2} \mathrm{O}$ (Table 3, entry 4) in $\mathrm{CDCl}_{3}$. The peak at $\delta 4.65 \mathrm{ppm}$ is assigned to the methine protons of repeated units in PCHC as well as the peaks at $\delta 3.56$ and 4.37 are assigned to methine protons that is adjacent to end-capped hydroxyl and carbonate in PCHC, respectively. 


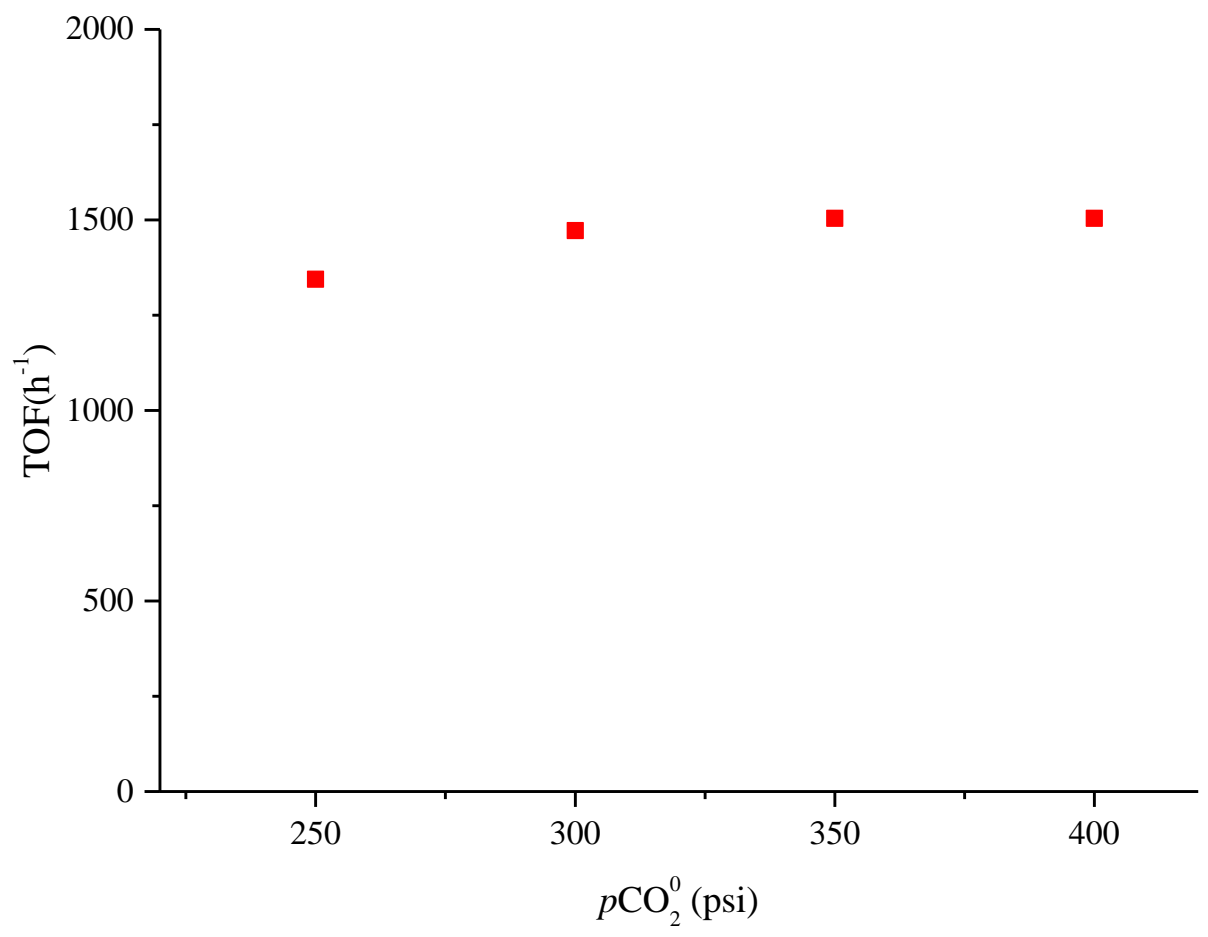

Figure S12. The plot of TOFs with varying initial $\mathrm{CO}_{2}$ pressures employing dinickel complex $1(0.03125 \mathrm{~mol} \%)$ at $140{ }^{\circ} \mathrm{C}$ for $1 \mathrm{~h}$. 


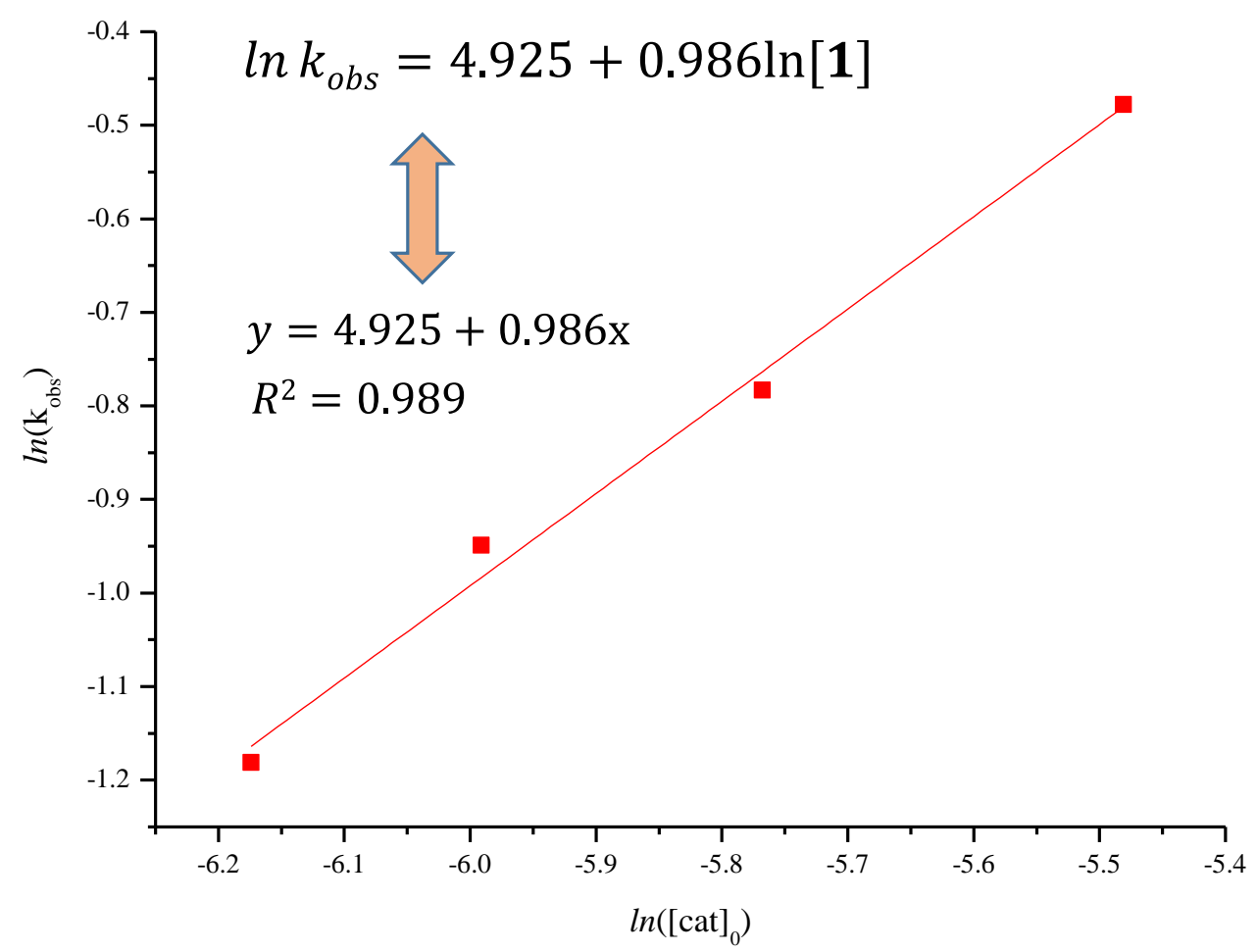

Figure S13. A linear plot of $\ln k_{\mathrm{obs}}$ versus $\ln [1]_{0}$ exhibiting a first-order dependence on dinickel catalyst concentration. All polymerizations were performed at $140{ }^{\circ} \mathrm{C}$ and an initial $\mathrm{CO}_{2}$ pressure of 300 psi. 
Table S1. Copolymerization of $\mathrm{CO}_{2}$ and $\mathrm{CHO}$ Catalyzed by Utilizing Dinickel Complex 1 under Different Initial $\mathrm{CO}_{2}$ Pressures for $1 \mathrm{~h}$

\begin{tabular}{|c|c|c|c|c|c|}
\hline Entry & $\begin{array}{l}\text { Pressure } \\
\text { /psi }\end{array}$ & $\begin{array}{l}\% \mathrm{CHO} \\
\text { Conv. }^{a}\end{array}$ & $\begin{array}{c}\% \text { Copolymer }^{a} \\
\left(\% \mathrm{CO}_{3} \text { linkages }\right)^{b}\end{array}$ & $\mathrm{TON}^{c}$ & $\begin{array}{l}\text { TOF } \\
/ \mathrm{h}^{-1 d}\end{array}$ \\
\hline S1 & 250 & 42 & $>99(>99)$ & 1344 & 1344 \\
\hline S2 & 300 & 46 & $>99(>99)$ & 1472 & 1472 \\
\hline S3 & 350 & 47 & $>99(>99)$ & 1504 & 1504 \\
\hline $\mathrm{S} 4$ & 400 & 47 & $>99(>99)$ & 1504 & 1504 \\
\hline
\end{tabular}

Copolymerization conditions: $0.03125 \mathrm{~mol} \%$ catalyst, $100.0 \mathrm{mmol} \mathrm{CHO}, \mathrm{T}=140{ }^{\circ} \mathrm{C} .{ }^{a}$ Based on ${ }^{1} \mathrm{H}$ NMR analysis of the reaction mixture. ${ }^{b}$ Based on ${ }^{1} \mathrm{H}$ NMR determination of the purified copolymers. ${ }^{c} \mathrm{TON}=$ number of moles of $\mathrm{CHO}$ consumed per mole of catalyst. ${ }^{d} \mathrm{TOF}=\mathrm{TON}$ per hour. 
Table S2. Kinetic Studies of $\mathrm{CO}_{2} / \mathrm{CHO}$ Copolymerization Catalyzed by Dinickel Complex 1 at Various Monomer-to-Catalyst Ratio $(2400,3200,4000 \text { and 4800 })^{a}$

\begin{tabular}{|c|c|c|c|c|}
\hline Entry & {$[\mathrm{CHO}]_{0}:[\mathbf{1}]_{0}$} & Time/min & $\%$ CHO Conv. ${ }^{b}$ & $\ln \left([\mathrm{CHO}]_{0} /[\mathrm{CHO}]_{\mathrm{t}}\right)$ \\
\hline $\mathrm{S} 1$ & 2400 & 15 & 30 & 0.357 \\
\hline $\mathrm{S} 2$ & 2400 & 30 & 38 & 0.478 \\
\hline $\mathrm{S} 3$ & 2400 & 45 & 47 & 0.635 \\
\hline S4 & 2400 & 60 & 56 & 0.821 \\
\hline S5 & 3200 & 20 & 26 & 0.301 \\
\hline S6 & 3200 & 30 & 33 & 0.400 \\
\hline S7 & 3200 & 60 & 46 & 0.616 \\
\hline S8 & 3200 & 90 & 57 & 0.844 \\
\hline S9 & 4000 & 30 & 15 & 0.163 \\
\hline $\mathrm{S} 10$ & 4000 & 45 & 21 & 0.236 \\
\hline S11 & 4000 & 60 & 28 & 0.329 \\
\hline $\mathrm{S} 12$ & 4000 & 90 & 42 & 0.545 \\
\hline S13 & 4800 & 30 & 10 & 0.105 \\
\hline S14 & 4800 & 60 & 21 & 0.236 \\
\hline S15 & 4800 & 90 & 33 & 0.400 \\
\hline S16 & 4800 & 120 & 43 & 0.562 \\
\hline
\end{tabular}

Copolymerization conditions: $100.0 \mathrm{mmol} \mathrm{CHO}, 140{ }^{\circ} \mathrm{C}, \mathrm{pCO}_{2}{ }^{0}=300 \mathrm{psi}, 1 \mathrm{~h} .{ }^{a} \mathrm{All}$ runs displayed excellent copolymerization selectivity (>99\% carbonate linkages) based on ${ }^{1} \mathrm{H}$ NMR determination. ${ }^{b}$ Based on ${ }^{1} \mathrm{H}$ NMR analysis of the reaction mixture. 
Table S3. Kinetic Parameters for $\mathrm{CO}_{2} / \mathrm{CHO}$ Copolymerization Catalyzed by Dinickel Complex 1 at Different Catalyst concentrations

\begin{tabular}{|c|c|c|c|c|c|}
\hline Entry & {$[\mathrm{CHO}]_{0}:[\mathbf{1}]_{0}$} & $\begin{array}{l}\text { Observed rate coefficient, } \\
\qquad k_{\mathrm{obs}}\left(\mathrm{M}^{-1} \mathrm{~h}^{-1}\right)^{a}\end{array}$ & $\ln \left(k_{\mathrm{obs}}\right)$ & $\begin{array}{l}\text { catalyst concentrations } \\
\qquad[\mathbf{1}](\mathbf{M})^{b}\end{array}$ & $\ln [1]$ \\
\hline $\mathrm{S} 1$ & 2400 & 0.620 & -0.478 & $4.167 \times 10^{-3}$ & -5.481 \\
\hline $\mathrm{S} 2$ & 3200 & 0.457 & -0.783 & $3.125 \times 10^{-3}$ & -5.768 \\
\hline $\mathrm{S} 3$ & 4000 & 0.387 & -0.949 & $2.50 \times 10^{-3}$ & -5.991 \\
\hline $\mathrm{S} 4$ & 4800 & 0.307 & -1.181 & $2.08 \times 10^{-3}$ & -6.175 \\
\hline
\end{tabular}

${ }^{a}$ Calculated from the slope of the fitted regression line of Figure 6.

${ }^{b}$ A fixed amount of CHO (100.0 mmol) was used. 
Table S4. Crystallographic Data of Complexes 1-9

\begin{tabular}{|c|c|c|c|}
\hline & 1 & 2 & 3 \\
\hline formula & $\mathrm{C}_{52} \mathrm{H}_{64} \mathrm{~F}_{6} \mathrm{~N}_{8} \mathrm{Ni}_{2} \mathrm{O}_{6}$ & $\mathrm{C}_{38} \mathrm{H}_{36} \mathrm{~F}_{6} \mathrm{~N}_{8} \mathrm{Ni}_{2} \mathrm{O}_{6}$ & $\mathrm{C}_{64} \mathrm{H}_{72} \mathrm{~F}_{6} \mathrm{~N}_{8} \mathrm{Ni}_{2} \mathrm{O}_{6}$ \\
\hline Formula weight & 1128.53 & 932.17 & 1280.71 \\
\hline Temp (K) & $202 \mathrm{~K}$ & $150(2) \mathrm{K}$ & $150.0 \mathrm{~K}$ \\
\hline Crystal system & Monoclinic & Monoclinic & Triclinic \\
\hline Space group & $P 21 / \mathrm{c}$ & $C 2 / \mathrm{c}$ & $P-1$ \\
\hline $\mathrm{a}(\AA)$ & $14.4449(15)$ & 25.999(3) & $12.3500(14)$ \\
\hline $\mathrm{b}(\AA)$ & $33.109(3)$ & $12.2433(13)$ & $14.3025(15)$ \\
\hline$c(\AA)$ & $11.9977(11)$ & $26.190(3)$ & $21.079(2)$ \\
\hline$\alpha(\operatorname{deg})$ & 90 & 90 & $97.269(4)$ \\
\hline$\beta(\operatorname{deg})$ & $110.809(4)$ & $104.573(5)$ & $102.969(4)^{\circ}$ \\
\hline$\gamma(\mathrm{deg})$ & 90 & 90 & $97.252(4)$ \\
\hline$V\left(\AA^{3}\right)$ & $5363.7(9)$ & $8068.4(16)$ & $3552.8(7)$ \\
\hline $\mathrm{Z}$ & 4 & 8 & 2 \\
\hline$D_{\text {calc }}\left(\mathrm{Mg} / \mathrm{m}^{3}\right)$ & 1.398 & 1.535 & 1.197 \\
\hline$\mu(\operatorname{Mo~K} \alpha)\left(\mathrm{mm}^{-1}\right)$ & 0.778 & 1.017 & 0.596 \\
\hline$F(000)$ & 2360 & 3824 & 1340 \\
\hline Reflections collected & 83391 & 62852 & 48888 \\
\hline No. of parameters & 692 & 545 & 787 \\
\hline Indep. reflns $\left(R_{\text {int }}\right)$ & $11070(0.0765)$ & $7007(0.1021)$ & $12448(0.0565)$ \\
\hline$R 1[I>2 \sigma(I)]$ & 0.0436 & 0.0489 & 0.0833 \\
\hline $\mathrm{w} R 2[I>2 \sigma(I)]$ & 0.1004 & 0.1152 & 0.2573 \\
\hline Goodness-of-fit on $F^{2}$ & 1.044 & 1.028 & 1.073 \\
\hline
\end{tabular}


Table S4. Crystallographic Data of Complexes 1-9 (Cont'd)

\begin{tabular}{|c|c|c|c|}
\hline & 4 & 5 & 6 \\
\hline formula & $\mathrm{C}_{50} \mathrm{H}_{43} \mathrm{~F}_{6} \mathrm{~N}_{8} \mathrm{Ni}_{2} \mathrm{O}_{6}$ & $\mathrm{C}_{62} \mathrm{H}_{74} \mathrm{~N}_{8} \mathrm{Ni}_{2} \mathrm{O}_{6}$ & $\mathrm{C}_{48} \mathrm{H}_{48} \mathrm{~N}_{8} \mathrm{Ni}_{2} \mathrm{O}_{7}$ \\
\hline Formula weight & 1083.34 & 1144.71 & 966.36 \\
\hline Temp (K) & $150(2) \mathrm{K}$ & $175(2) \mathrm{K}$ & $153(2) \mathrm{K}$ \\
\hline Crystal system & Monoclinic & Monoclinic & Monoclinic \\
\hline Space group & $P 21 / \mathrm{n}$ & $C 2 / \mathrm{c}$ & $P 21 / \mathrm{c}$ \\
\hline $\mathrm{a}(\AA)$ & $14.5120(8)$ & $33.843(2)$ & $15.5150(7)$ \\
\hline $\mathrm{b}(\AA)$ & $22.4483(12)$ & $12.7808(8)$ & $18.2972(8)$ \\
\hline$c(\AA)$ & $17.5820(10)$ & $32.0586(17)$ & $16.6085(7)$ \\
\hline$\alpha(\operatorname{deg})$ & 90 & 90 & 90 \\
\hline$\beta(\operatorname{deg})$ & $102.676(2)$ & $110.996(3)$ & $109.937(2)$ \\
\hline$\gamma(\operatorname{deg})$ & 90 & 90 & 90 \\
\hline$V\left(\AA^{3}\right)$ & $5588.1(5)$ & $12946.0(13)$ & $4432.3(3)$ \\
\hline $\mathrm{Z}$ & 4 & 8 & 4 \\
\hline$D_{\text {calc }}\left(\mathrm{Mg} / \mathrm{m}^{3}\right)$ & 1.288 & 1.175 & 1.448 \\
\hline$\mu(\operatorname{Mo~K} \alpha)\left(\mathrm{mm}^{-1}\right)$ & 0.745 & 0.633 & 0.912 \\
\hline$F(000)$ & 2228 & 4848 & 2016 \\
\hline Reflections collected & 87538 & 88470 & 60651 \\
\hline No. of parameters & 658 & 715 & 591 \\
\hline Indep. reflns $\left(R_{\text {int }}\right)$ & $11358(0.0311)$ & $11323(0.0200)$ & $7781(0.0726)$ \\
\hline$R 1[I>2 \sigma(I)]$ & 0.0451 & 0.0347 & 0.0487 \\
\hline $\mathrm{w} R 2[I>2 \sigma(I)]$ & 0.1261 & 0.0914 & 0.0941 \\
\hline Goodness-of-fit on $F^{2}$ & 1.055 & 1.154 & 3.067 \\
\hline
\end{tabular}


Table S4. Crystallographic Data of Complexes 1-9 (Cont'd)

\begin{tabular}{|c|c|c|c|}
\hline & 7 & 8 & 9 \\
\hline formula & $\mathrm{C}_{64} \mathrm{H}_{79} \mathrm{~N}_{8} \mathrm{Ni}_{2} \mathrm{O}_{9}$ & $\mathrm{C}_{50} \mathrm{H}_{52} \mathrm{~N}_{8} \mathrm{Ni}_{2} \mathrm{O}_{9}$ & $\mathrm{C}_{48} \mathrm{H}_{64} \mathrm{~N}_{10} \mathrm{Ni}_{2} \mathrm{O}_{8}$ \\
\hline Formula weight & 1221.77 & 1026.41 & 1026.51 \\
\hline Temp (K) & $150(2)$ & $150(2)$ & $150.15 \mathrm{~K}$ \\
\hline Crystal system & Orthorhombic & Monoclinic & Monoclinic \\
\hline Space group & Pbca & $P 21 / \mathrm{c}$ & $P 21 / \mathrm{c}$ \\
\hline $\mathrm{a}(\AA)$ & $26.6316(17)$ & $16.2422(7)$ & 17.621(3) \\
\hline $\mathrm{b}(\AA)$ & $16.9726(11)$ & $18.3555(8)$ & 23.683(4) \\
\hline$c(\AA)$ & $30.280(2)$ & $16.9507(7)$ & $16.353(3)$ \\
\hline$\alpha(\operatorname{deg})$ & 90 & 90 & 90 \\
\hline$\beta(\operatorname{deg})$ & 90 & $110.649(2)$ & $113.073(6)$ \\
\hline$\gamma(\operatorname{deg})$ & 90 & 90 & 90 \\
\hline$V\left(\AA^{3}\right)$ & $13686.8(15)$ & $4728.9(4)$ & $6278.5(19)$ \\
\hline $\mathrm{Z}$ & 8 & 4 & 4 \\
\hline$D_{\text {calc }}\left(\mathrm{Mg} / \mathrm{m}^{3}\right)$ & 1.186 & 1.442 & 1.086 \\
\hline$\mu(\operatorname{Mo~K\alpha })\left(\mathrm{mm}^{-1}\right)$ & 0.606 & 0.863 & 0.649 \\
\hline$F(000)$ & 5176 & 2144 & 2168 \\
\hline Reflections collected & 185354 & 73726 & 84157 \\
\hline No. of parameters & 763 & 629 & 625 \\
\hline Indep. reflns $\left(R_{\text {int }}\right)$ & $12027(0.1132)$ & $9649(0.0478)$ & $11015(0.0666)$ \\
\hline$R 1[I>2 \sigma(I)]$ & 0.0789 & 0.0385 & 0.0776 \\
\hline $\mathrm{w} R 2[I>2 \sigma(I)]$ & 0.2690 & 0.1144 & 0.2475 \\
\hline Goodness-of-fit on $F^{2}$ & 1.051 & 0.810 & 1.089 \\
\hline
\end{tabular}

